\title{
The Low-velocity, Rapidly Fading Type la Supernova 2002es
}

\section{Citation}

Ganeshalingam, Mohan, Weidong Li, Alexei V. Filippenko, Jeffrey M. Silverman, Ryan Chornock, Ryan J. Foley, Thomas Matheson, et al. 2012. "THE LOW-VELOCITY, RAPIDLY FADING TYPE la SUPERNOVA 2002es." The Astrophysical Journal 751 (2): 142. https:// doi.org/10.1088/0004-637x/751/2/142.

\section{Permanent link}

http://nrs.harvard.edu/urn-3:HUL.InstRepos:41399798

\section{Terms of Use}

This article was downloaded from Harvard University's DASH repository, and is made available under the terms and conditions applicable to Other Posted Material, as set forth at http:// nrs.harvard.edu/urn-3:HUL.InstRepos:dash.current.terms-of-use\#LAA

\section{Share Your Story}

The Harvard community has made this article openly available. Please share how this access benefits you. Submit a story.

Accessibility 


\title{
THE LOW-VELOCITY, RAPIDLY FADING TYPE Ia SUPERNOVA 2002es
}

\author{
Mohan Ganeshalingam ${ }^{1}$, Weidong Li ${ }^{1,6}$, Alexei V. Filippenko ${ }^{1}$, Jeffrey M. Silverman ${ }^{1}$, Ryan Chornock ${ }^{2}$, \\ Ryan J. Foley ${ }^{2,7}$, Thomas Matheson ${ }^{3}$, Robert P. Kirshner ${ }^{2}$, Peter Milne ${ }^{4}$, Mike Calkins ${ }^{2}$, and Ken J. Shen ${ }^{1,5,8}$ \\ ${ }^{1}$ Department of Astronomy, University of California, Berkeley, CA 94720-3411, USA; mganesh @ astro.berkeley.edu \\ ${ }^{2}$ Harvard-Smithsonian Center for Astrophysics, 60 Garden Street, Cambridge, MA 02138, USA \\ ${ }^{3}$ National Optical Astronomy Observatory, 950 North Cherry Avenue, Tucson, AZ 85719, USA \\ ${ }^{4}$ Steward Observatory, University of Arizona, 933 North Cherry Avenue, Tucson, AZ 85721, USA \\ ${ }^{5}$ Lawrence Berkeley National Laboratory, Berkeley, CA 94720, USA \\ Received 2012 February 8; accepted 2012 March 31; published 2012 May 16
}

\begin{abstract}
SN 2002es is a peculiar subluminous Type Ia supernova (SN Ia) with a combination of observed characteristics never before seen in an SN Ia. At maximum light, SN 2002es shares spectroscopic properties with the underluminous SN $1991 \mathrm{bg}$ subclass of SNe Ia, but with substantially lower expansion velocities $\left(\sim 6000 \mathrm{~km} \mathrm{~s}^{-1}\right)$ more typical of the peculiar SN 2002cx subclass. Photometrically, SN 2002es differs from both SN 1991bg-like and SN 2002cx-like supernovae. Although at maximum light it is subluminous $\left(M_{B}=-17.78 \mathrm{mag}\right)$, SN 2002es has a relatively broad light curve $\left(\Delta m_{15}(B)=1.28 \pm 0.04 \mathrm{mag}\right)$, making it a significant outlier in the light-curve width versus luminosity relationship. We estimate a ${ }^{56} \mathrm{Ni}$ mass of $0.17 \pm 0.05 M_{\odot}$ synthesized in the explosion, relatively low for an SN Ia. One month after maximum light, we find an unexpected plummet in the bolometric luminosity. The late-time decay of the light curves is inconsistent with our estimated ${ }^{56} \mathrm{Ni}$ mass, indicating that either the light curve was not completely powered by ${ }^{56} \mathrm{Ni}$ decay or the ejecta became optically thin to $\gamma$-rays within a month after maximum light. The host galaxy is classified as an S0 galaxy with little to no star formation, indicating that the progenitor of SN 2002es is likely from an old stellar population. We also present a less extensive data set for SN 1999bh, an object which shares similar photometric and spectroscopic properties. Both objects were found as part of the Lick Observatory Supernova Search, allowing us to estimate that these objects should account for $2.5 \%$ of SNe Ia within a fixed volume. Current theoretical models are unable to explain the observed characteristics of SN 2002es.
\end{abstract}

Key words: supernovae: general - supernovae: individual (SN 2002es, SN 1999bh)

Online-only material: color figures

\section{INTRODUCTION}

Type Ia supernovae (SNe Ia) are the runaway thermonuclear explosions of carbon-oxygen white dwarfs (WDs). SNe Ia are characterized spectroscopically by an absence of hydrogen and the presence of intermediate-mass elements (IMEs; e.g., silicon, sulfur, oxygen) and iron-group elements (iron, cobalt) (e.g., Filippenko 1997 and references therein). A majority of spectroscopically identified SNe Ia form a class of objects with a standardizable luminosity, allowing for their use as accurate distance indicators. Application of SNe Ia on extragalactic scales led to the discovery that the universe is accelerating in its expansion (Riess et al. 1998; Perlmutter et al. 1999). Subsequent application of large samples of SNe Ia out to high redshifts (WoodVasey et al. 2007; Hicken et al. 2009b; Kessler et al. 2009; Amanullah et al. 2010; Sullivan et al. 2011a; Suzuki et al. 2012) has led to precise estimates of cosmological parameters when combined with measurements of baryon acoustic oscillations and anisotropy in the cosmic microwave background (CMB).

The cosmological application of SNe Ia is predicated on the relationship between the peak absolute magnitude of an $\mathrm{SN}$, the width of its light curve, and its color. Phillips (1993) found that $\mathrm{SNe}$ with slowly declining light curves had a larger luminosity at maximum light. Applying corrections for light-curve width and SN color (as a measurement of host-galaxy extinction and intrinsic scatter in $\mathrm{SN}$ colors) has allowed SNe Ia to be accurate distance indicators to within $10 \%$ in distance (Jha et al. 2007;

\footnotetext{
6 Deceased 2011 December 12.

7 Clay Fellow.

8 Einstein Fellow.
}

Guy et al. 2007; Conley et al. 2008). There are indications that including spectral information (Foley et al. 2008; Wang et al. 2009a; Bailey et al. 2009; Foley \& Kasen 2011; Blondin et al. 2011; Silverman et al. 2012a) and host-galaxy information (Kelly et al. 2010; Sullivan et al. 2010; Lampeitl et al. 2010) further improves distance estimates.

Despite the ability to standardize the luminosity of SNe Ia based on observed light-curve properties, a significant fraction of SN Ia events have peculiar characteristics, including some overluminous and underluminous objects (e.g., Filippenko 1997 and references therein). In particular, Filippenko et al. (1992) and Leibundgut et al. (1993) found that optical light curves of SN 1991bg evolved rapidly, and its peak luminosity was $\sim 2 \mathrm{mag}$ fainter than that of normal objects. Moreover, the maximumlight spectrum of SN 1991bg showed strong Ti II absorption, indicating a relatively cool photosphere, and the expansion velocity at maximum light as measured from the absorption minimum in the blueshifted Si II $\lambda 6355$ feature was $\sim 10,000 \mathrm{~km} \mathrm{~s}^{-1}$, slightly lower than expansion velocities measured for normal SNe Ia ( 11,000-13,000 $\mathrm{km} \mathrm{s}^{-1}$; Filippenko et al. 1992). Since the initial identification of SN 1991bg as a subclass of SNe Ia, many members belonging to the subclass have been identified by the SN community. Studies of the host-galaxy morphology indicate that SN 1991bg-like objects are found preferentially in early-type galaxies (Howell 2001; Ganeshalingam et al. 2010; $\mathrm{Li}$ et al. 2011a), leading to the suggestion that the progenitors of SN 1991bg-like SNe come from old stellar populations. There is currently debate about the cosmological utility of SN 1991bglike objects as standardizable candles (Jha et al. 2007; Guy et al. 2007). 
More recently, a range of properties for peculiar subluminous SNe Ia has been discovered. Foley et al. (2010b) presented evidence that SN 2006bt spectroscopically resembled SN 1991bg, but photometrically resembled a normal SN Ia. SN 2006bt was discovered at a projected distance of $33.7 \mathrm{kpc}$ from the nucleus of its early-type host galaxy. The Palomar Transient Factory (PTF; Law et al. 2009) has published data on two peculiar subluminous objects, both discovered at large distances from the likely host galaxy. PTF 09dav was an abnormally subluminous SN Ia $\left(M_{B}=-15.44 \mathrm{mag}\right)$ with a narrow light curve found $41 \mathrm{kpc}$ from its host galaxy (Sullivan et al. 2011b). Maguire et al. (2011) presented data on the subluminous PTF 10ops which shared many similarities with SN 2006bt. PTF 10ops had a broad light curve and was seen at a projected distance of $148 \mathrm{kpc}$ from the nominal host. All objects had spectral features that match those of SN 1991bg, although PTF 09dav had particularly slow expansion velocities of $6100 \mathrm{~km} \mathrm{~s}^{-1}$.

Objects similar to SN 2002cx (Filippenko 2003; Li et al. 2003b) form another subclass of subluminous peculiar SNe Ia. These objects have maximum-light spectra similar to those of overluminous objects like SN 1991T, characterized by weak Si II $\lambda 6355$ features and dominated by Fe III lines, indicating a hot photosphere. However, the expansion velocities of these objects at maximum light are $\sim 6000 \mathrm{~km} \mathrm{~s}^{-1}$, indicating an explosion with low kinetic energy per unit mass. There appears to be a great diversity among SN 2002cx-like objects, with a distribution of absolute luminosity and kinetic energy (Narayan et al. 2011; McClelland et al. 2010). SN 2008ha is the faintest member of the subclass, with $M_{V}=-14.2 \mathrm{mag}$ and velocities of $\sim 4000-5000 \mathrm{~km} \mathrm{~s}^{-1}$ at maximum light (Foley et al. 2010a).

Here we report our observations of SN 2002es, an object somewhat spectroscopically similar to $\mathrm{SN} 1991 \mathrm{bg}$, further adding to the puzzle of subluminous SNe Ia. SN 2002es was discovered (Li et al. 2002) on unfiltered CCD images at $\sim 16.3 \mathrm{mag}$ as part of the Lick Observatory Supernova Search (LOSS) with the $0.76 \mathrm{~m}$ Katzman Automatic Imaging Telescope (KAIT; Filippenko et al. 2001; Li et al. 2003a) on 2002 August 23.5 (UT dates are used throughout this paper) in UGC 2708. Its J2000 coordinates are $\alpha=03^{\mathrm{h}} 23^{\mathrm{m}} 47^{\mathrm{s}} .23$ and $\delta=+40^{\circ} 33^{\prime} 53^{\prime \prime} .5$, which is $19^{\prime \prime} \mathrm{W}$ and $26^{\prime \prime} \mathrm{N}$ of the galaxy nucleus ( $\mathrm{Li}$ et al. 2002). Subsequent optical spectroscopic observations on 2002 September 3 by Chornock et al. (2002) and Matheson et al. (2002) classified SN 2002es as an SN 1991bg-like object near maximum light based on the presence of strong Ti II, Si II, and $\mathrm{O}_{\text {II }}$ absorption features, but with an expansion velocity of $\sim 6000 \mathrm{~km} \mathrm{~s}^{-1}$ as measured from the absorption minimum of the Si II $\lambda 6355$ feature. This is notably lower than typical expansion velocities of $\sim 11,000 \mathrm{~km} \mathrm{~s}^{-1}$ found in normal SNe Ia and SN 1991bg-like objects and more typical of SN 2002cxlike objects ( $\mathrm{Li}$ et al. 2003b).

Noting the peculiarity of SN 2002es, our group started a photometric and spectroscopic campaign to document the evolution of this unique object. Here, we present $B V R I$ photometry for SN 2002es starting a week before maximum light collected as part of the LOSS SN Ia photometry program (Ganeshalingam et al. 2010). Late-time photometry was obtained with the $2.3 \mathrm{~m}$ Bok telescope at Steward Observatory on Kitt Peak in Arizona to constrain the late-time decay of the light curve. We also show an extensive optical spectral series covering the evolution of SN 2002es from maximum light to two months after maximum. In addition, we argue that SN 1999bh is an SN 2002es-like event.

The rest of the paper is structured as follows. Our data and reduction techniques are presented in Section 2, with a detailed

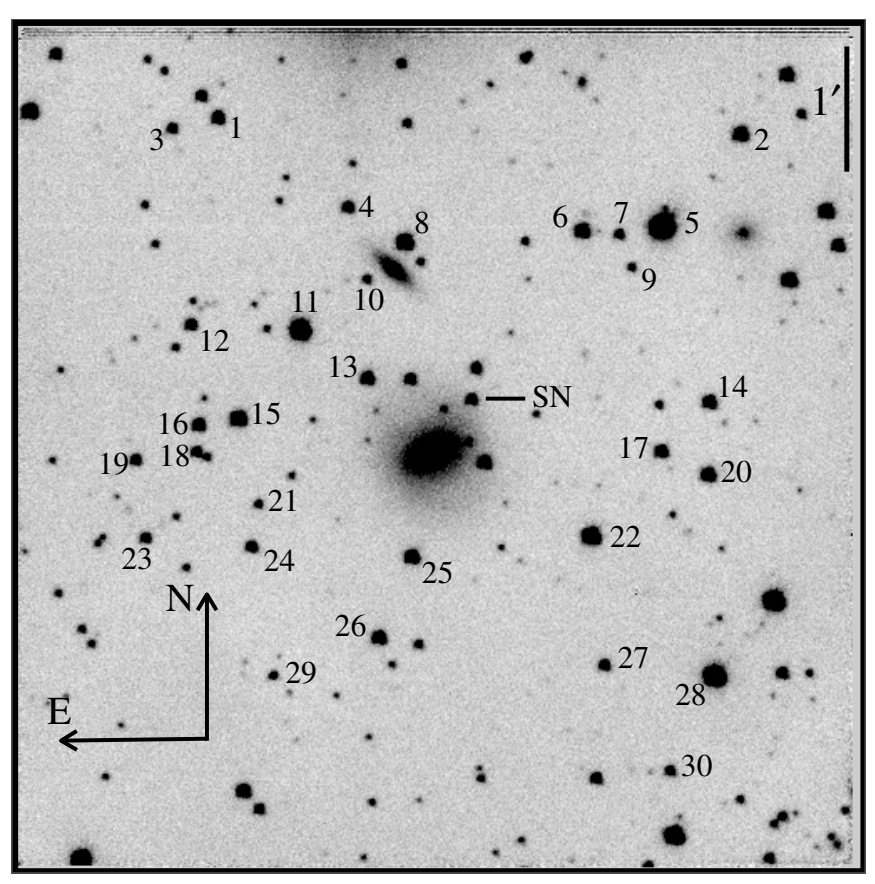

Figure 1. KAIT $V$-band image of SN 2002es. The field is $6.7 \times 6.7$. The SN and comparison stars are marked. The labels of the comparison stars correspond to the numbers in Table 1.

analysis of our photometry and spectroscopy in Section 3. In Section 4, we discuss possible physical interpretations for the observed properties of SN 2002es and compare SN 2002es to theoretical models. We summarize our results in Section 5.

\section{OBSERVATIONS}

\subsection{Photometry}

Broadband photometric follow-up observations of SN 2002es started on 2002 August 24.48 (one week before $B_{\max }$ ) with KAIT in the BVRI bands. SN 2002es was monitored with a 1-2 day cadence for the first month after discovery, resulting in well-sampled light curves. Additional $B V R I$ photometry from the $1 \mathrm{~m}$ Nickel telescope at Lick Observatory was obtained to complement the KAIT data. Late-time data in $B V R$ were taken using the $2.3 \mathrm{~m}$ Bok telescope. A $V$-band image of the field from KAIT is shown in Figure 1.

Images were bias subtracted and flat fielded using standard procedures in IRAF. ${ }^{9} \mathrm{SN} 2002 \mathrm{es}$ was sufficiently close to its host galaxy that galaxy subtraction was required to disentangle SN light from galaxy light. Templates for the field were obtained for the KAIT, Nickel, and Bok telescopes after SN 2002es faded beyond the detection limit of each telescope ( $>2 \mathrm{yr}$ after discovery) and subtracted from the data images. The flux from the SN was measured in comparison to local field stars using point-spread function (PSF) fitting photometry. The uncertainties in our photometric measurements were estimated by randomly injecting artificial stars with the same PSF and magnitude as the $\mathrm{SN}$ into the data images. The images with artificial stars were rerun through our galaxy-subtraction and photometry routines, and the scatter in doing this 20 times was adopted as the photometric measurement error.

\footnotetext{
9 The Image Reduction and Analysis Facility (IRAF) is distributed by the National Optical Astronomy Observatory, which is operated by the

Association of Universities for Research in Astronomy, Inc., under cooperative agreement with the National Science Foundation.
} 
Table 1

Photometry of Local Standard Stars

\begin{tabular}{|c|c|c|c|c|c|c|c|}
\hline ID & $\begin{array}{c}\alpha \\
(\mathrm{J} 2000)\end{array}$ & $\begin{array}{c}\delta \\
(\mathrm{J} 2000)\end{array}$ & $\begin{array}{l}B\left(\sigma_{B}\right) \\
(\mathrm{mag})\end{array}$ & $\begin{array}{l}V\left(\sigma_{V}\right) \\
(\mathrm{mag})\end{array}$ & $\begin{array}{l}R\left(\sigma_{R}\right) \\
(\mathrm{mag})\end{array}$ & $\begin{array}{l}I\left(\sigma_{I}\right) \\
(\mathrm{mag})\end{array}$ & $N_{\text {calib }}$ \\
\hline 1 & $03: 23: 57.81$ & $+40: 36: 08.0$ & $17.403(011)$ & $16.497(008)$ & $15.940(012)$ & $15.462(007)$ & 4 \\
\hline 2 & $03: 23: 35.94$ & $+40: 36: 00.3$ & $16.470(010)$ & $15.675(007)$ & $15.206(007)$ & $14.744(012)$ & 5 \\
\hline 3 & $03: 23: 59.75$ & $+40: 36: 02.8$ & $17.817(010)$ & $17.174(011)$ & $16.785(012)$ & $16.331(014)$ & 3 \\
\hline 4 & $03: 23: 52.38$ & $+40: 35: 25.2$ & $17.440(010)$ & $16.844(007)$ & $16.482(011)$ & $16.104(011)$ & 4 \\
\hline 5 & $03: 23: 39.24$ & $+40: 35: 16.1$ & 14.293 (009) & $12.933(014)$ & $12.191(002)$ & $11.545(011)$ & 2 \\
\hline 6 & $03: 23: 42.58$ & $+40: 35: 14.0$ & $16.442(007)$ & $15.702(003)$ & $15.265(005)$ & $14.851(011)$ & 5 \\
\hline 7 & $03: 23: 41.02$ & $+40: 35: 12.2$ & $17.978(011)$ & $17.314(011)$ & $16.905(013)$ & $16.482(010)$ & 5 \\
\hline 8 & $03: 23: 50.00$ & $+40: 35: 08.6$ & $15.777(011)$ & $15.119(005)$ & $14.727(007)$ & $14.325(011)$ & 4 \\
\hline 9 & $03: 23: 40.51$ & $+40: 34: 56.4$ & $18.618(013)$ & $17.970(004)$ & $17.579(010)$ & $17.035(008)$ & 2 \\
\hline 10 & $03: 23: 51.59$ & $+40: 34: 50.8$ & $18.657(013)$ & $17.786(006)$ & $17.292(008)$ & $16.865(006)$ & 3 \\
\hline 11 & $03: 23: 54.37$ & $+40: 34: 26.8$ & $14.523(012)$ & $14.040(011)$ & $13.736(013)$ & $13.407(006)$ & 4 \\
\hline 12 & $03: 23: 58.96$ & $+40: 34: 29.2$ & $17.496(012)$ & $16.721(011)$ & $16.254(013)$ & $15.735(013)$ & 4 \\
\hline 13 & $03: 23: 51.57$ & $+40: 34: 03.6$ & $16.938(010)$ & $16.180(005)$ & $15.744(011)$ & $15.296(015)$ & 4 \\
\hline 14 & $03: 23: 37.25$ & $+40: 33: 52.3$ & $17.115(009)$ & $16.126(006)$ & $15.542(007)$ & $15.047(012)$ & 4 \\
\hline 15 & $03: 23: 56.96$ & $+40: 33: 44.5$ & $15.783(012)$ & $15.258(012)$ & $14.905(007)$ & $14.554(010)$ & 4 \\
\hline 16 & $03: 23: 58.62$ & $+40: 33: 41.4$ & $16.993(010)$ & $16.280(011)$ & $15.833(008)$ & $15.395(015)$ & 4 \\
\hline 17 & $03: 23: 39.26$ & $+40: 33: 28.8$ & $16.795(010)$ & $16.222(005)$ & $15.863(008)$ & $15.503(012)$ & 4 \\
\hline 18 & $03: 23: 58.71$ & $+40: 33: 28.4$ & $17.935(011)$ & $17.021(003)$ & $16.477(008)$ & $16.003(007)$ & 4 \\
\hline 19 & $03: 24: 01.27$ & $+40: 33: 24.7$ & $18.038(013)$ & $16.979(010)$ & $16.402(008)$ & $15.814(017)$ & 3 \\
\hline 20 & $03: 23: 37.30$ & $+40: 33: 17.8$ & $16.652(013)$ & $15.842(011)$ & $15.360(009)$ & $14.935(013)$ & 4 \\
\hline 21 & $03: 23: 56.13$ & $+40: 33: 03.5$ & $18.860(010)$ & $17.906(005)$ & $17.374(012)$ & $16.956(008)$ & 3 \\
\hline 22 & $03: 23: 42.20$ & $+40: 32: 48.4$ & $15.821(011)$ & $14.705(003)$ & $14.090(005)$ & $13.512(009)$ & 5 \\
\hline 23 & $03: 24: 00.85$ & $+40: 32: 47.5$ & 17.769 (009) & $17.064(005)$ & $16.658(006)$ & $16.233(005)$ & 2 \\
\hline 24 & $03: 23: 56.43$ & $+40: 32: 43.2$ & $17.724(011)$ & $16.895(004)$ & 16.377 (009) & $15.836(013)$ & 5 \\
\hline 25 & $03: 23: 49.70$ & $+40: 32: 38.5$ & 16.919 (009) & $15.795(004)$ & $15.101(007)$ & $14.540(003)$ & 4 \\
\hline 26 & $03: 23: 51.07$ & $+40: 31: 60.0$ & $16.680(011)$ & $15.987(005)$ & $15.583(007)$ & $15.170(013)$ & 5 \\
\hline 27 & $03: 23: 41.64$ & $+40: 31: 46.7$ & $18.034(012)$ & $16.877(011)$ & $16.157(012)$ & $15.481(014)$ & 2 \\
\hline 28 & $03: 23: 37.03$ & $+40: 31: 41.7$ & $14.570(007)$ & $13.860(007)$ & $13.427(012)$ & $13.003(016)$ & 3 \\
\hline 29 & $03: 23: 55.50$ & $+40: 31: 41.6$ & $18.669(011)$ & $17.703(012)$ & $17.062(011)$ & $16.456(007)$ & 4 \\
\hline 30 & $03: 23: 38.90$ & $+40: 30: 56.3$ & $17.954(013)$ & $17.261(010)$ & $16.836(013)$ & $16.417(014)$ & 4 \\
\hline
\end{tabular}

Note. $1 \sigma$ uncertainties (in units of $0.001 \mathrm{mag}$ ) are listed in parentheses.

Instrumental magnitudes were color corrected to the Landolt system (Landolt 1992, 2009) using the average color terms measured from multiple photometric nights. The magnitudes of local field stars were calibrated against Landolt standards on six photometric nights. The results are reported in Table 1 . The final photometric error is the photometric measurement error added in quadrature with the error in the calibration of our field stars. The final photometry is presented in Table 2 .

In addition to the photometry presented here, we supplement our light curves with UBVRI data presented for SN 2002es in the CfA3 photometry sample (Hicken et al. 2009a) taken with the $1.2 \mathrm{~m}$ telescope at Fred Lawrence Whipple Observatory (FLWO) in Arizona operated by the Harvard Smithsonian Center for Astrophysics (CfA). The agreement between the KAIT and CfA3 data is within $\sim 0.05$ mag in all bands, with no evidence of a systematic offset. The light curves from all telescopes are presented in Figure 2.

\subsection{Spectroscopy}

Low-resolution spectra of SN 2002es were obtained using the Kast dual spectrograph mounted on the $3 \mathrm{~m}$ Shane Telescope at Lick Observatory (Miller \& Stone 1993), the FAST spectrograph mounted on the $1.5 \mathrm{~m}$ Tillinghast telescope at FLWO (Fabricant et al. 1998), and the Low Resolution Imaging Spectrometer on the $10 \mathrm{~m}$ Keck I telescope (LRIS; Oke et al. 1995). All of our observations were taken at the optimal parallactic angle to minimize differential light loss (Filippenko 1982).
All spectra of SN 2002es were reduced using standard CCD processing techniques (e.g., Foley et al. 2003; Matheson et al. 2008). Processing and extraction of the one-dimensional spectra were performed in IRAF using the optimal extraction algorithm of Horne (1986). The wavelength calibration was obtained by fitting low-order polynomials to calibration-lamp spectra. Our spectra were flux calibrated using our own IDL routines. Corrections for telluric absorption features were made using spectrophotometric standards (Wade \& Horne 1988) taken at roughly the same airmass as the $\mathrm{SN}$ observation. Table 3 presents a summary of our spectroscopic observations.

\subsection{Host Galaxy}

The NASA/IPAC Extragalactic Database (NED) ${ }^{10}$ lists two discordant values for the heliocentric redshift of UGC 2708 from different sources. The NED Web site for UGC 2708 lists $z_{\text {helio }}=0.028$ as the default redshift determined from marginal measurements of the H I $21 \mathrm{~cm}$ line by Monnier Ragaigne et al. (2003). NED warns under the "Essential Note" section that de Vaucouleurs et al. (1991) find $z_{\text {helio }}=0.018$ from optical lines. We obtained a spectrum of UGC 2708 with Kast at Lick Observatory on 2006 July 21.4 to determine the actual redshift of the host galaxy. We find a heliocentric redshift of $z_{\text {hel }}=$ $0.0182 \pm 0.0001$ from measurements of weak, narrow $\mathrm{H} \alpha+$ [N II] emission lines, in good agreement with the value of de Vaucouleurs et al. (1991). The heliocentric redshift of UGC 2708

\footnotetext{
10 http://ned.ipac.caltech.edu/.
} 
Table 2

Photometry of SN 2002es

\begin{tabular}{|c|c|c|c|c|c|}
\hline $\mathrm{JD}-2,452,000$ & $\begin{array}{c}B \\
(\mathrm{mag})\end{array}$ & $\begin{array}{c}V \\
(\mathrm{mag})\end{array}$ & $\begin{array}{c}R \\
(\mathrm{mag})\end{array}$ & $\begin{array}{c}I \\
(\mathrm{mag})\end{array}$ & Telescope \\
\hline 510.98 & $17.682(033)$ & $17.245(028)$ & $17.007(025)$ & $16.835(031)$ & KAIT \\
\hline 511.98 & $17.625(029)$ & $17.148(027)$ & $16.882(022)$ & $16.711(029)$ & KAIT \\
\hline 512.97 & $17.498(033)$ & $17.050(028)$ & $16.786(027)$ & $16.622(030)$ & KAIT \\
\hline 514.00 & $17.482(034)$ & $17.009(029)$ & $16.711(029)$ & $16.521(033)$ & KAIT \\
\hline 515.01 & $17.453(035)$ & $16.918(026)$ & $16.669(020)$ & $16.470(030)$ & KAIT \\
\hline 516.01 & $\ldots$ & $16.752(111)$ & $16.552(097)$ & $16.375(033)$ & KAIT \\
\hline 517.02 & $17.372(104)$ & $\ldots$ & $16.587(055)$ & $16.347(046)$ & KAIT \\
\hline 518.01 & $17.307(044)$ & $16.778(045)$ & $16.465(090)$ & $16.374(022)$ & KAIT \\
\hline 519.02 & $17.373(020)$ & $16.817(024)$ & $16.503(020)$ & $16.332(026)$ & KAIT \\
\hline 520.01 & $17.369(020)$ & $16.762(020)$ & $16.493(022)$ & $16.318(026)$ & KAIT \\
\hline 520.99 & $17.414(026)$ & $16.790(020)$ & $16.453(020)$ & $16.303(026)$ & KAIT \\
\hline 521.99 & $\ldots$ & $16.829(031)$ & $\ldots$ & $16.211(111)$ & KAIT \\
\hline 523.02 & $17.506(023)$ & $16.829(021)$ & $16.484(020)$ & $16.320(031)$ & KAIT \\
\hline 524.02 & $17.550(026)$ & $16.844(020)$ & $16.498(020)$ & $16.339(037)$ & KAIT \\
\hline 524.95 & $17.729(020)$ & $16.925(020)$ & $16.514(020)$ & $16.311(020)$ & Nickel \\
\hline 525.95 & $17.805(020)$ & $16.985(020)$ & $16.539(020)$ & $16.336(020)$ & Nickel \\
\hline 528.97 & $18.094(020)$ & $17.165(020)$ & $16.687(020)$ & $16.400(020)$ & Nickel \\
\hline 529.00 & $18.142(030)$ & $17.164(028)$ & $16.640(023)$ & $16.400(023)$ & KAIT \\
\hline 530.01 & $18.255(040)$ & $17.193(025)$ & $16.746(020)$ & $16.446(023)$ & KAIT \\
\hline 531.01 & $18.323(039)$ & $17.293(031)$ & $16.774(020)$ & $16.492(022)$ & KAIT \\
\hline 531.99 & $18.500(049)$ & $17.354(020)$ & $16.814(020)$ & $16.533(029)$ & KAIT \\
\hline 533.00 & $18.583(071)$ & $17.525(080)$ & $16.914(105)$ & $16.588(101)$ & KAIT \\
\hline 535.00 & $18.779(037)$ & $17.556(031)$ & $16.977(033)$ & $16.621(025)$ & KAIT \\
\hline 536.02 & $18.824(053)$ & $17.629(032)$ & $17.042(033)$ & $16.641(028)$ & KAIT \\
\hline 537.99 & $18.876(094)$ & $17.717(050)$ & $17.122(030)$ & $16.732(043)$ & KAIT \\
\hline 540.87 & $19.041(155)$ & $17.836(063)$ & $17.248(043)$ & $16.792(058)$ & KAIT \\
\hline 547.98 & $19.429(167)$ & $18.275(084)$ & $17.715(036)$ & $17.110(055)$ & KAIT \\
\hline 550.97 & $19.610(082)$ & $18.464(061)$ & $17.910(032)$ & $17.346(033)$ & KAIT \\
\hline 551.99 & $19.744(032)$ & $18.570(025)$ & $17.964(020)$ & $17.417(042)$ & Nickel \\
\hline 552.84 & $19.771(043)$ & $18.631(027)$ & $18.010(021)$ & $17.505(025)$ & Nickel \\
\hline 553.96 & $19.834(100)$ & $18.602(070)$ & $18.116(038)$ & $17.615(058)$ & KAIT \\
\hline 561.91 & $\cdots$ & $\cdots$ & $\cdots$ & $18.481(334)$ & KAIT \\
\hline 562.91 & $\cdots$ & $19.447(118)$ & 19.167 (109) & $18.508(123)$ & KAIT \\
\hline 566.87 & $\ldots$ & $19.579(161)$ & $19.445(125)$ & $18.961(120)$ & KAIT \\
\hline 573.86 & $\cdots$ & $20.155(171)$ & $\cdots$ & $\cdots$ & KAIT \\
\hline 577.92 & $\cdots$ & $20.811(307)$ & $\cdots$ & $\cdots$ & KAIT \\
\hline 592.89 & $23.180(150)$ & $22.711(110)$ & $22.34(110)$ & $\ldots$ & Bok \\
\hline
\end{tabular}

Note. $1 \sigma$ uncertainties (in units of $0.001 \mathrm{mag}$ ) are listed in parentheses.

Table 3

Log of Optical Spectral Observations for SN 2002es

\begin{tabular}{|c|c|c|c|c|c|}
\hline UT Date & $\begin{array}{l}\text { Phase }^{\mathrm{a}} \\
\text { (days) }\end{array}$ & Telescope/Instrument & $\begin{array}{c}\text { Exposure Time } \\
\text { (s) }\end{array}$ & $\begin{array}{c}\text { Res. }^{\mathrm{b}} \\
(\AA)\end{array}$ & Observer $^{\mathrm{c}}$ \\
\hline 2002 Sep 03.5 & +3 & Lick/Kast & 600 & $5-12$ & $\mathrm{AF}, \mathrm{RC}, \mathrm{BS}$ \\
\hline 2002 Sep 05.5 & +5 & FLWO/FAST & 1200 & $6-7$ & $\mathrm{MC}$ \\
\hline 2002 Sep 06.5 & +6 & FLWO/FAST & 1200 & $6-7$ & $\mathrm{MC}$ \\
\hline 2002 Sep 10.5 & +10 & FLWO/FAST & 1200 & $6-7$ & PB \\
\hline 2002 Sep 12.5 & +12 & FLWO/FAST & 1200 & $6-7$ & $\mathrm{MC}$ \\
\hline 2002 Sep 13.3 & +13 & Lick/Kast & 1200 & $5-12$ & RF, SJ, MP \\
\hline 2002 Sep 28.4 & +28 & FLWO/FAST & 1200 & $6-7$ & $\mathrm{MC}$ \\
\hline 2002 Oct 01.3 & +30 & Lick/Kast & 1800 & $5-12$ & $\mathrm{AF}, \mathrm{RF}$ \\
\hline 2002 Oct 8 & +37 & Keck I/LRIS & 400 & $6-7$ & $\mathrm{AF}, \mathrm{RC}$ \\
\hline 2002 Nov 8 & +67 & Keck I/LRIS & 1800 & $6-7$ & $\mathrm{AF}, \mathrm{RC}, \mathrm{SJ}, \mathrm{BB}$ \\
\hline 2002 Nov 11 & +70 & Keck I/LRIS & 1800 & $6-7$ & $\mathrm{AF}, \mathrm{RC}$ \\
\hline
\end{tabular}

Notes.

${ }^{\text {a }}$ Rest-frame days relative to the date of $B_{\max }, 2002$ August 31.8 (JD 2,452,518.3), rounded to the nearest day.

b Approximate spectral resolution.

c AF: A. Filippenko; BB: B. Barris; BS: B. Swift; MC: M. Calkins; MP: M. Papenkova; PB: P. Berlind; RC: R. Chornock; RF: R. Foley; SJ: S. Jha. 


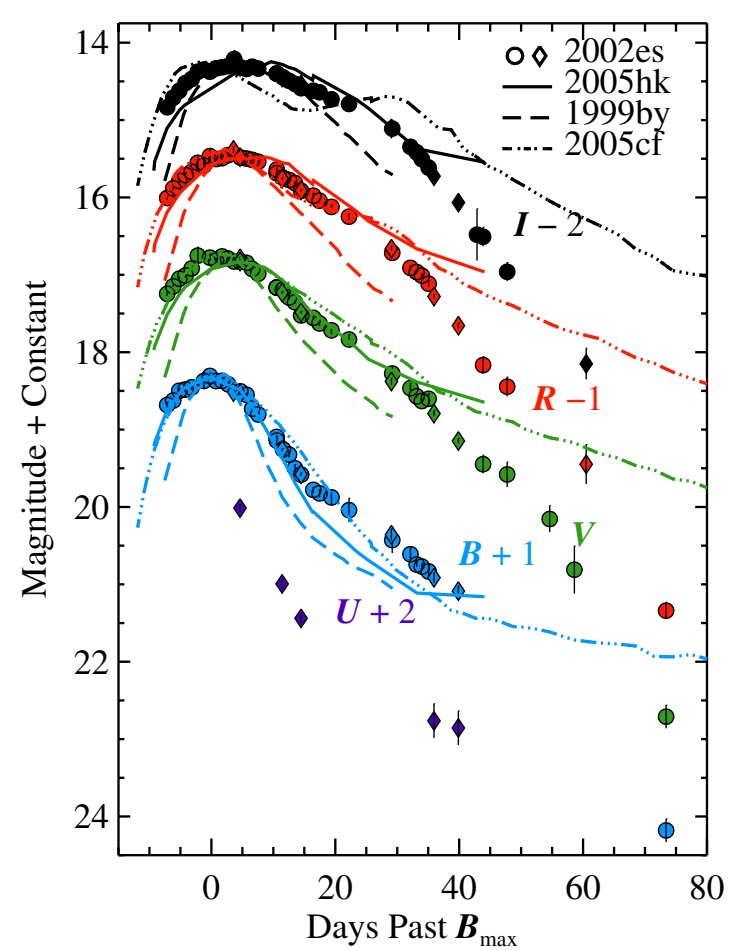

Figure 2. UBVRI light curves of SN 2002es. Data from the KAIT, Nickel, and Bok telescopes are plotted as circles. Data from Hicken et al. (2009a) are shown as diamonds. For comparison, we include the SN 2002cx-like SN 2005hk (solid line), the SN 1991bg-like SN 1999by (dashed), and the normal SN 2005cf (dotdashed line). Comparison light curves have been shifted to have the same peak magnitude and phase as SN 2002es. Note the particularly fast decline in all bands at $t>+30$ days compared to the other objects.

(A color version of this figure is available in the online journal.)

corresponds to $z_{\mathrm{CMB}}=0.0177$ (in the frame of the $\mathrm{CMB}$ ). We adopt an uncertainty of $300 \mathrm{~km} \mathrm{~s}^{-1}$ to account for any peculiar motions induced by gravitational interactions with neighboring galaxies. For a standard $\Lambda$ CDM cosmology with $\Omega_{\mathrm{m}}=0.27$, $\Omega_{\Lambda}=0.73, w=-1$, and $H_{0}=73.8 \mathrm{~km} \mathrm{~s}^{-1} \mathrm{Mpc}^{-1}$ (Riess et al. 2011), we find a luminosity distance $d_{L}=73.20 \pm 4.41 \mathrm{Mpc}$ and a distance modulus $\mu=34.32 \pm 0.12 \mathrm{mag}$. SN 2002es exploded at a projected distance of $11 \mathrm{kpc}$ from the nucleus of UGC 2708 .

The original IAUCs that spectroscopically classify SN 2002es (Chornock et al. 2002; Matheson et al. 2002) predate the source for the incorrect redshift listed on NED and likely used the correct redshift supplied by de Vaucouleurs et al. (1991). The correct redshift for SN 2002es was also used in the cosmology analysis of Hicken et al. (2009b).

UGC 2708 was included in a study of SN Ia host-galaxy properties by Neill et al. (2009). The authors estimated galaxy properties by fitting template-galaxy spectral energy distributions (SEDs) to multi-wavelength photometry from the Sloan Digital Sky Survey (SDSS) and the Galaxy Evolution Explorer. For UGC 2708, the authors estimated a minimal amount of active star formation and $E(B-V)_{\text {host }}=0.0 \mathrm{mag}$. This finding, along with the absence of $\mathrm{Na}$ I $\mathrm{D}$ absorption at the redshift of the host galaxy in spectra of SN 2002es, indicates that the reddening due to the host galaxy is negligible.

UGC 2708 was also observed spectroscopically as part of SDSS. Using the publicly available line-flux measurements, we find an $[\mathrm{N}$ II $] / \mathrm{H} \alpha$ ratio consistent with that of a low-ionization nuclear emission-line region (LINER; Heckman 1980) or a composite galaxy. Coupled with the results from Neill et al.
Table 4

Photometric Properties of SN 2002es

\begin{tabular}{lcccc}
\hline \hline Filter & JD of Max $-2,452,000$ & Mag at Max $^{\mathrm{a}}$ & Peak Abs. Mag. & $\begin{array}{c}\Delta m_{15} \\
(\mathrm{mag})\end{array}$ \\
\hline$B$ & $518.30 \pm 0.28$ & $17.33 \pm 0.02$ & $-17.78 \pm 0.12$ & $1.28 \pm 0.04$ \\
$V$ & $519.21 \pm 0.28$ & $16.78 \pm 0.02$ & $-18.15 \pm 0.12$ & $0.74 \pm 0.02$ \\
$R$ & $520.96 \pm 0.30$ & $16.47 \pm 0.02$ & $-18.35 \pm 0.12$ & $0.57 \pm 0.02$ \\
$I$ & $521.39 \pm 0.33$ & $16.29 \pm 0.02$ & $-18.40 \pm 0.12$ & $0.37 \pm 0.02$ \\
\hline
\end{tabular}

Notes.

a Not corrected for Milky Way or host-galaxy extinction.

${ }^{\mathrm{b}}$ Corrected for Milky Way extinction and assuming no host-galaxy extinction.

(2009), UGC 2708 is likely a LINER with no active star formation.

\section{RESULTS}

\subsection{Photometry}

The light curves of SN 2002es are displayed in Figure 2, along with light curves of the normal SN Ia 2005cf (Wang et al. 2008; Ganeshalingam et al. 2010), the SN 2002cx-like SN Ia 2005hk (Phillips et al. 2007), and the SN 1991bg-like SN Ia 1999by (Ganeshalingam et al. 2010).

Basic photometric properties for the light curves of SN 2002es are reported in Table 4. All values were measured using a fifth-order polynomial fit directly to the data. Uncertainties were estimated using a Monte Carlo routine to produce 50 realizations of our data set. Each individual data set realization was produced by randomly perturbing each photometry data point using its photometric uncertainty assuming a Gaussian distribution. Light-curve properties were measured for each realization, and the final measurements were found by taking the mean and standard deviation of the set of simulated realizations.

The light curves of SN 2002es share some characteristics in common with the subluminous SN 1991bg subtype. SN 2002es lacks a prominent shoulder in the $R$ and $I$ bands (Filippenko et al. 1992; Leibundgut et al. 1993). The secondary maximum often found in $R$ and $I$ (and more prominently in the near-infrared) is attributed to the cooling of the ejecta to temperatures where the transition from Fe III to Fe II becomes favorable, redistributing flux from shorter wavelengths to longer wavelengths (Kasen 2006). This transition occurs earlier in cooler SNe. From models of the radiative transfer within SNe, Kasen (2006) finds that the timing and strength of the shoulder is dependent on the distribution and amount of ${ }^{56} \mathrm{Ni}$ within the ejecta. Models with a completely homogenized composition and with a small amount of ${ }^{56} \mathrm{Ni}$ result in an $I$-band light curve with no discernible secondary peak or shoulder. Instead, the two peaks merge to produce a single broad peak. Given the similar absolute magnitudes of SN 2002es and SN 1991bg, the lack of a shoulder or secondary maximum in the $R$ and $I$ bands may be a consequence of low ${ }^{56} \mathrm{Ni}$ production, if the light curve is powered by the decay of ${ }^{56} \mathrm{Ni}$.

The timing of maximum light in each band is similar to that of SN 1991bg-like SNe. In normal SNe, maximum light in the $I$ band precedes that of $B$ by a few days (as evident in the light curves of SN 2005cf in Figure 2). Taubenberger et al. (2008) find that for the SN 1991bg-like SN 2005bl, peak brightness in $U B V R I$ occurred in successive order of bluest to reddest filter with the date of maximum light in each band separated by $\sim 1$ day, similar to what is seen in SN 2002es. 


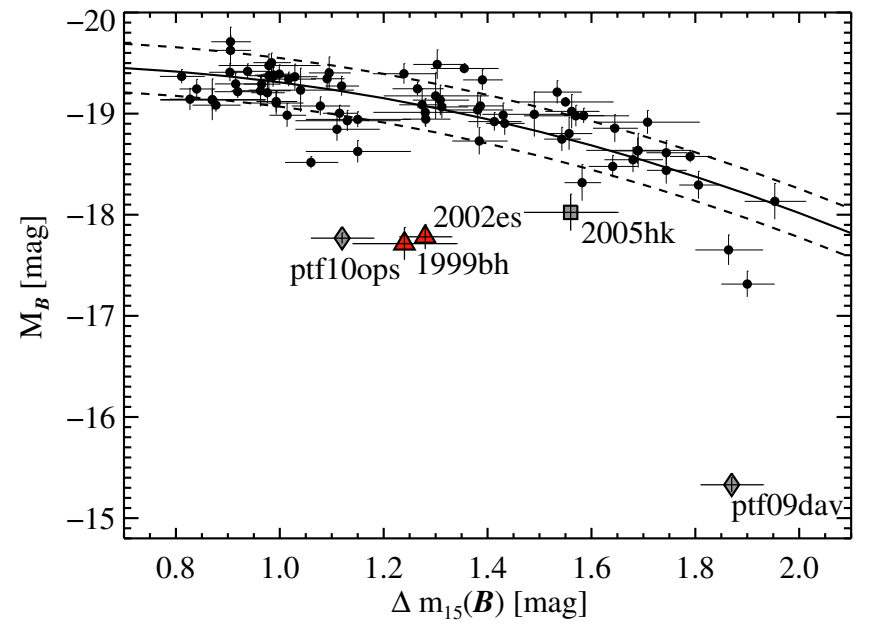

Figure 3. $M_{B}$ as a function of $\Delta m_{15}(B)$ for $76 \mathrm{SNe}$ from Ganeshalingam et al. (2010) corrected for host-galaxy extinction using values derived from the MLCS distance-fitting software. Overplotted as a solid line is the quadratic Phillips relation from Phillips et al. (1999) shifted along the ordinate to match the data. The dashed lines correspond to the $1 \sigma$ scatter about the relationship. SN 2002es and SN 1999bh (red triangles) are clear outliers in the relationship. For comparison, we include other subluminous peculiar objects SN 2005hk, PTF 09dav, and PTF 10ops.

(A color version of this figure is available in the online journal.)

The $B$-band peak magnitude of $\mathrm{SN} 2002$ es is $17.33 \pm$ $0.02 \mathrm{mag}$ after correcting for a color excess of $E(B-V)=$ 0.183 mag from Milky Way extinction (Schlegel et al. 1998). We assume negligible host-galaxy extinction based on the classification of UGC 2708 as an S0 galaxy and no evidence of Na I D absorption at the host-galaxy redshift. This corresponds to an absolute magnitude of $M_{B}=-17.78 \pm 0.12 \mathrm{mag}$, comparable to other subluminous SNe Ia, but brighter than SN 1991bg-like objects (Taubenberger et al. 2008). The absolute magnitude of SN 2002es is brighter than that of the SN 1991bg-like SN 2005bl by $\sim 0.5-1 \mathrm{mag}$ in $B V R I$.

Despite these similarities with SN 1991bg-like SNe Ia, the light curves of SN 2002es are significantly broader in all bands compared to those of most subluminous objects. Using the decline in magnitudes between maximum light and 15 days after maximum light in the $B$ band as a proxy for light-curve width (Phillips 1993), we measure $\Delta m_{15}(B)=1.28 \pm 0.04$ mag. Other SN 1991 bg-like SNe typically have $\Delta m_{15}(B) \approx 1.9$ mag (Taubenberger et al. 2008).

The light curves of SN 2002es also share similarities to the SN 2002cx-like SN 2005hk. Both objects are subluminous events compared to normal SNe Ia with light curves that are broader than those of SN 1991bg-like objects and lack a shoulder in the $R$ and $I$ bands. SN 2002es has a slower $B$-band decline than most SN 2002cx-like objects. SN 2005hk had $\Delta m_{15}(B)=1.56 \pm 0.09$ mag.

As the outer ejecta turn transparent, the light curve is expected to be powered by the thermalization of $\gamma$-rays produced by the decay of ${ }^{56} \mathrm{Co}(\sim 50$ days after explosion). This is typically observed as a linear fading in all bands. Leibundgut (2000) finds typical decay rates for SNe Ia of 0.014 mag day $^{-1}$ in $B$, $0.028 \mathrm{mag} \mathrm{day}^{-1}$ in $V$, and $0.042 \mathrm{mag} \mathrm{day}^{-1}$ in $I$. For SN $1991 \mathrm{bg}$, the $B$-band decline is marginally faster at $0.019 \mathrm{mag} \mathrm{day}^{-1}$ and slower in $I$ at $0.040 \mathrm{mag}^{\mathrm{day}}{ }^{-1}$. The $V$-band decline has been found to be fairly constant among normal and SN 1991bg-like SNe Ia (Leibundgut 2000).

Using data at $t>+30$ days (relative to maximum light in the $B$ band), we measure a decline of $0.040 \pm 0.004$ mag day $^{-1}$ in $B$,

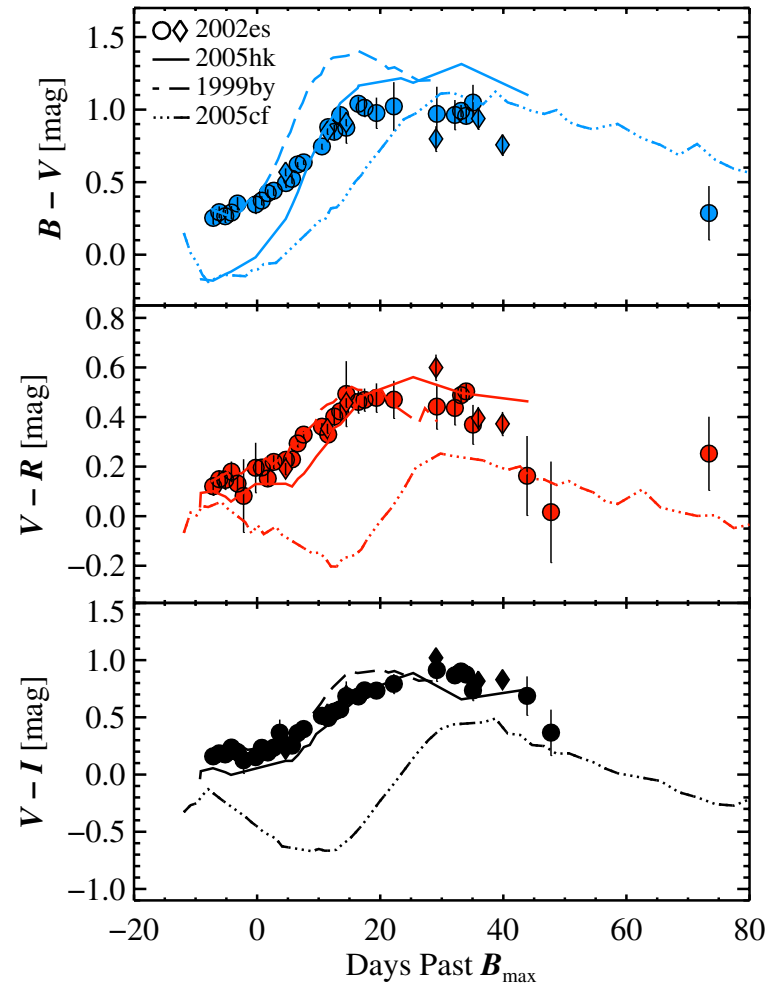

Figure 4. Optical color curves of $\mathrm{SN} 2002 \mathrm{es}$ corrected for $E(B-V)=$ 0.183 mag from Milky Way extinction. Solid circles are data taken with the KAIT and Nickel telescopes and solid diamonds are from Hicken et al. (2009a). Shown for comparison are SN 2005hk, SN 1999by, and SN 2005cf corrected for extinction using the reddening values provided in their respective reference. SN 2002es is significantly redder in all colors compared to SN 2005cf. None of the comparison color curves provides an adequate match to SN 2002es.

(A color version of this figure is available in the online journal.)

$0.081 \pm 0.004$ mag day $^{-1}$ in $V, 0.101 \pm 0.004$ mag day $^{-1}$ in $R$, and $0.099 \pm 0.004 \mathrm{mag} \mathrm{day}^{-1}$ in $I$. These rates are substantially faster than expected for an object powered by the decay of ${ }^{56} \mathrm{Co}$, even after accounting for the declining $\gamma$-ray deposition function as the ejecta expand homologously, and cast doubt on whether SN 2002es is necessarily a thermonuclear explosion. In Section 4.3, we discuss possible explanations for such a fast decline.

In Figure 3, we plot $\Delta m_{15}(B)$ versus $M_{B}$ for SN 2002es along with $76 \mathrm{SNe}$ taken from Ganeshalingam et al. (2010) using host-galaxy extinction values determined with the Multicolor Light-Curve Shape method (MLCS2k2.v006; Jha et al. 2007). The light-curve width versus luminosity relationship from Phillips et al. (1999), adjusted to $H_{0}=73.8 \mathrm{~km} \mathrm{~s}^{-1} \mathrm{Mpc}^{-1}$, is overplotted as a solid line with $1 \sigma$ scatter about the relation indicated by dashed lines. We also include measurements for the peculiar SNe SN 2005hk (Phillips et al. 2007), PTF 09dav (Sullivan et al. 2011b), and PTF 10ops (Maguire et al. 2011). SN 2002es is an obvious outlier $(5 \sigma)$ in the Phillips relation which predicts that SN 2002es should be $\sim 1.3$ mag brighter than the observed peak magnitude. The amount of host-galaxy extinction required to explain this discrepancy is unlikely given the absence of Na I D absorption in SN 2002es spectra at the redshift of the host galaxy.

\subsection{Color Curves}

In Figure 4, we plot the color evolution of SN 2002es along with SN 2005cf, SN 2005hk, and SN 1999by for comparison. All 


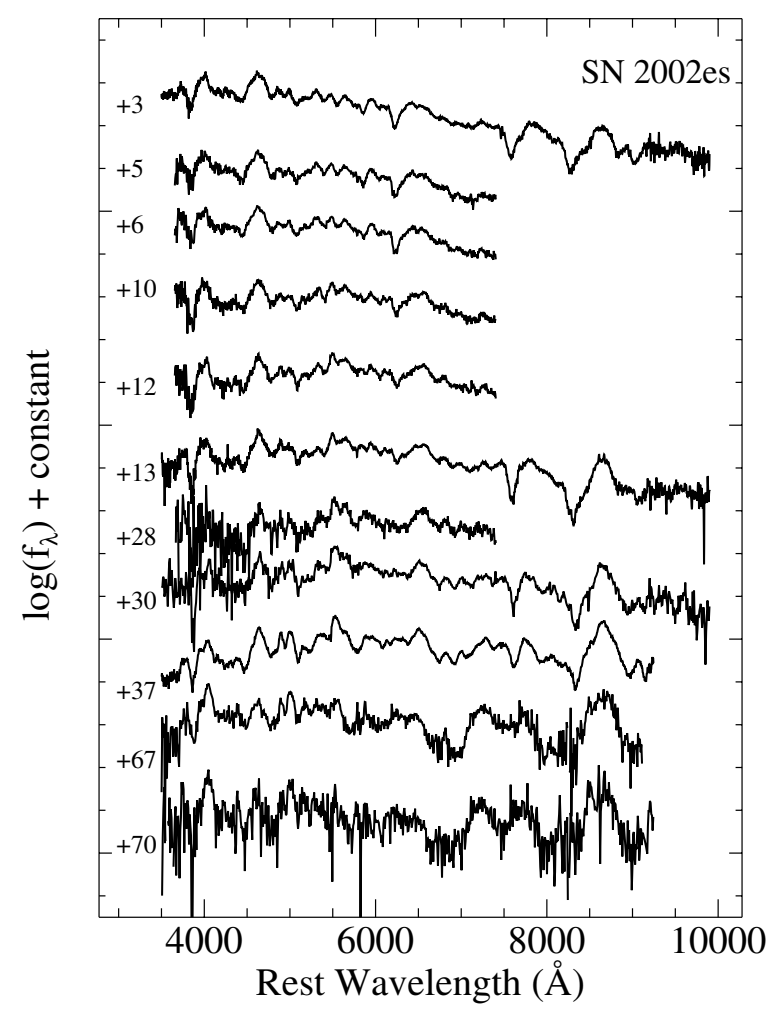

Figure 5. Montage of spectra of SN 2002es. All spectra have been shifted to the rest frame by the recession velocity of the host galaxy and corrected for $E(B-V)_{\mathrm{MW}}=0.183 \mathrm{mag}$. The phase relative to maximum light in the $B$ band is indicated to the left of each spectrum.

objects have been corrected for Milky Way extinction using the dust maps of Schlegel et al. (1998). We adopt a host-galaxy color excess of $E(B-V)_{\text {host }}=0.10 \mathrm{mag}$ for SN 2005cf (Wang et al. 2009b), $E(B-V)_{\text {host }}=0.10$ mag for SN 2005hk (Chornock et al. 2006), and $E(B-V)_{\text {host }}=0.0 \mathrm{mag}$ for SN 1999by (Garnavich et al. 2004). We assume no host-galaxy extinction for SN 2002es.

The $B-V$ color curve of SN 2002es does not match that of any of our comparison objects. The color evolution is most similar to that of the SN 1991bg-like SN 1999by, but with significant differences in $B-V$ after maximum light in $B$. Before $t\left(B_{\max }\right)$, SN 2002es and SN 1999by share a similar $B-V$ color evolution that is considerably redder than that of SN 2005cf and SN 2005hk. However, at $\sim 5$ day past maximum light in $B, \mathrm{SN} 1999$ by quickly becomes redder in $B-V$ while the color evolution of SN 2002es is much more gradual. The $V-R$ and $V-I$ color evolution for SN 2002es and SN 1999by appears much more similar. There is evidence that SN 2002es becomes bluer at $t>+35$ days, although the data are noisy.

\subsection{Spectral Properties}

In Figure 5, we show our time series of spectra covering the evolution of SN 2002es starting three days after maximum light in the $B$ band and extending out to two months after maximum light. The absence of $\mathrm{H} \alpha$ and the presence of Si II $\lambda 6355$ in earlytime spectra identify SN 2002es as an SN Ia (e.g., Filippenko 1997). Strong Ti II absorption around $4200 \AA$ is commonly associated with the subluminous SN 1991bg-like subclass of SNe Ia (Filippenko et al. 1992).

In Figure 6, we show our earliest spectrum of SN 2002es compared to spectra of the normal SN 2005cf (Wang et al. 2009b), the subluminous SN 1991bg (Turatto et al. 1996, via

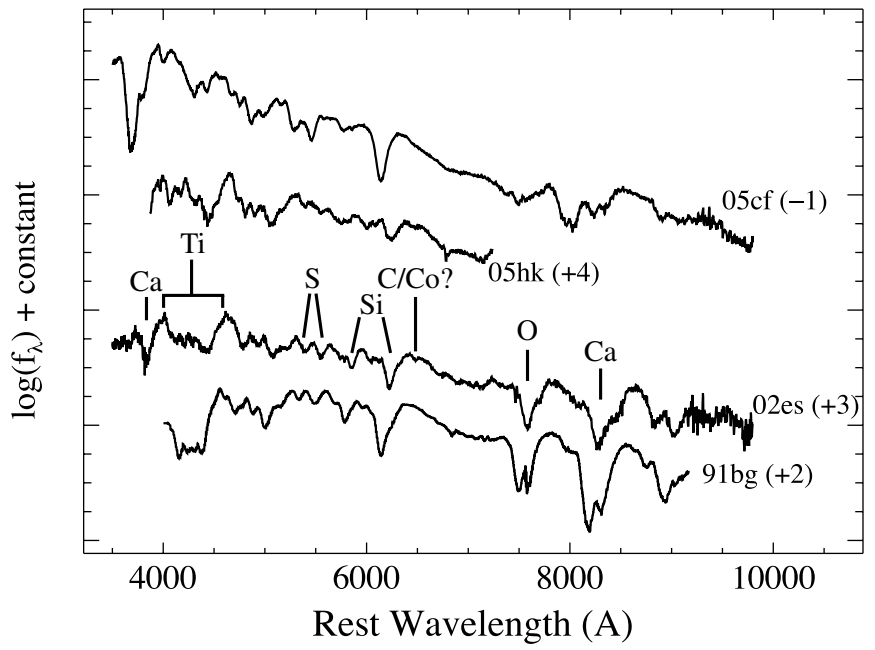

Figure 6. Spectrum of SN 2002es taken +3 days after maximum light in $B$, compared to the spectroscopically normal SN Ia 2005cf (Wang et al. 2009b), the SN 2002cx-like SN 2005hk (Phillips et al. 2007), and the subluminous SN 1991bg (Turatto et al. 1996) at similar phases. All objects have been shifted to the rest frame by their host-galaxy recession velocity and corrected for both Milky Way and host-galaxy extinction using the values in their respective references. Major spectroscopic features are identified. The spectrum of SN 2002es shares many similarities with SN 1991bg, except the former has significantly slower ejecta velocities. In particular, both objects show strong O I and Ti II features. The ejecta velocities of SN 2002es are more similar to those of SN $2005 \mathrm{hk}$.

the online SUSPECT database ${ }^{11}$ ), and the SN 2002cx-like SN 2005hk (Phillips et al. 2007). At this phase, SN 2002es shares the most similarities with SN 1991bg. However, the expansion velocity of the photosphere as measured from the minimum in the blueshift of the P-Cyngni profile of Si II $\lambda 6355$ is $6000 \mathrm{~km} \mathrm{~s}^{-1}$, significantly lower than typical values of $\sim 11,000 \mathrm{~km} \mathrm{~s}^{-1}$ for normal SNe Ia (Wang et al. 2009a; Foley et al. 2011) and SN 1991bg-like $\mathrm{SNe}$ of $\sim 10,000 \mathrm{~km} \mathrm{~s}^{-1}$ (Taubenberger et al. 2008). SN 2002cx-like objects have low ejecta velocities of $\sim 6000 \mathrm{~km} \mathrm{~s}^{-1}$ (Li et al. 2003b; Phillips et al. 2007), comparable to what is found in SN 2002es.

The spectrum of SN 2005hk only covers 3600-7400 A. Within this spectral range, it shows rather similar features to those of SN 2002es, but with notable differences as well. In particular, it has a weaker Si II $\lambda 6355$ absorption feature, and no obvious Ti II absorption trough near $4200 \AA$.

About two weeks after maximum light, SN 2002es begins to show more similarities with SN 2002cx-like objects. In Figure 7, we show our spectrum of SN 2002es from 13 days after maximum light compared to those of other objects at a similar phase. At this phase, Si II $\lambda 6355$ absorption is harder to discern. The Si II/Na I complex around $5700 \AA$ is weaker than what is seen in SN 1991bg. Similar to SN 2002cx-like objects, Fe II $\lambda \lambda 4555,5129$ absorption lines become more apparent and the $\mathrm{CaII} \mathrm{H} \& \mathrm{~K}$ lines are similar in strength. However, O I $\lambda 7774$ and the Ca II near-infrared (NIR) triplet are stronger in SN 2002es compared to SN 2005hk.

In Figure 8, we show our +37 day Keck spectrum along with comparison objects. We begin to see emission dominate in the case of the NIR Ca II triplet. The low expansion velocities in SN 2002es and SN 2005hk make it possible to see narrow features which are usually hard to discern due to the smearing of broad high-velocity features in normal SNe Ia. The spectrum is

\footnotetext{
11 http://suspect.nhn.ou.edu/ suspect.
} 


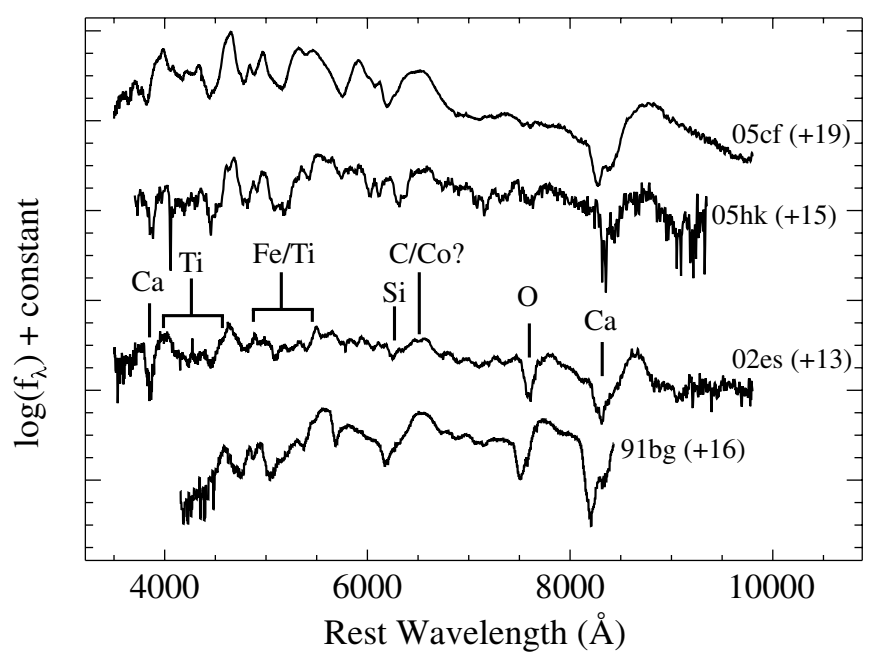

Figure 7. Spectrum of SN 2002es taken +13 days after maximum light. For comparison we show SN 2005cf (Wang et al. 2009b), SN 2005hk (Phillips et al. 2007), and SN 1991bg (Turatto et al. 1996) at a similar phase indicated in parentheses to the right of each spectrum. All spectra have been corrected for both Milky Way and host-galaxy extinction using the values in their respective references. Major spectroscopic features are identified.

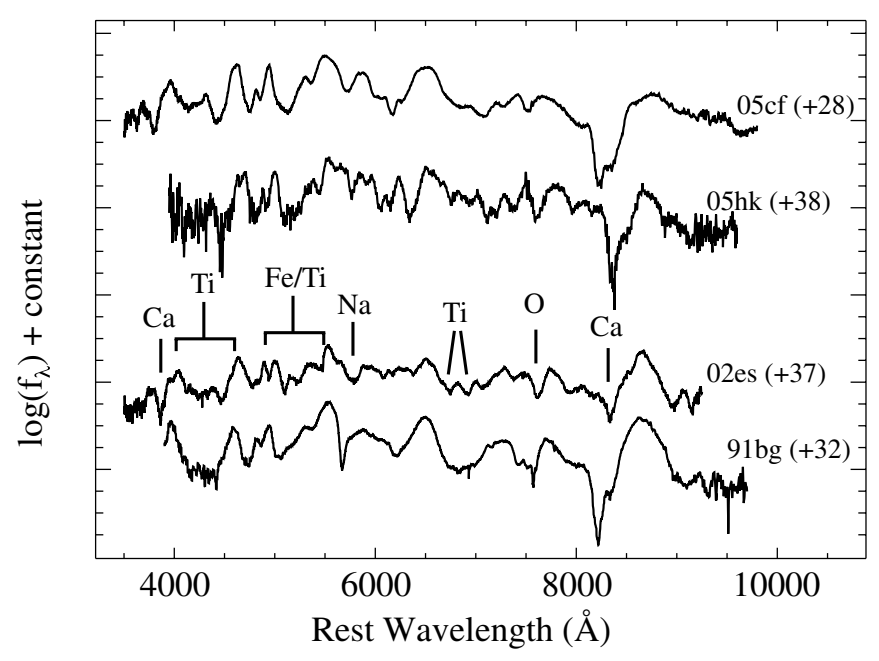

Figure 8. Spectrum of SN 2002es taken +37 days after maximum light using LRIS on Keck. For comparison we show SN 2005cf (Wang et al. 2009b), SN 2005hk (Phillips et al. 2007), and SN 1991bg (Turatto et al. 1996) at a similar phase indicated in parentheses. All spectra have been corrected for both Milky Way and host-galaxy extinction using the values in their respective references. Major spectroscopic features are identified.

dominated by permitted iron-group elements, but line blending makes it difficult to uniquely identify features.

We show our combined +67 day and +70 day spectrum of SN 2002es in Figure 9, along with spectra of the normal SN 1994D, SN 2005hk, and SN 1991bg at comparable phases. SN 2002es continues to share the most similarities with SN 1991bg. Despite the rapid evolution of the light curves, the spectra do not show signs of being completely nebular. We continue to see continuum emission and absorption in our combined spectrum. There is a hint of forbidden emission lines, possibly [Ca II] or [Fe II] in the region around $7200 \AA$, but we do not detect other prominent forbidden iron emission features commonly seen in nebular SN Ia spectra (Stanishev et al. 2007; Leloudas et al. 2009). We note that the permitted Ca II emission is not particularly strong, unlike PTF 09dav and the class of Ca-rich objects (Kasliwal et al. 2011).

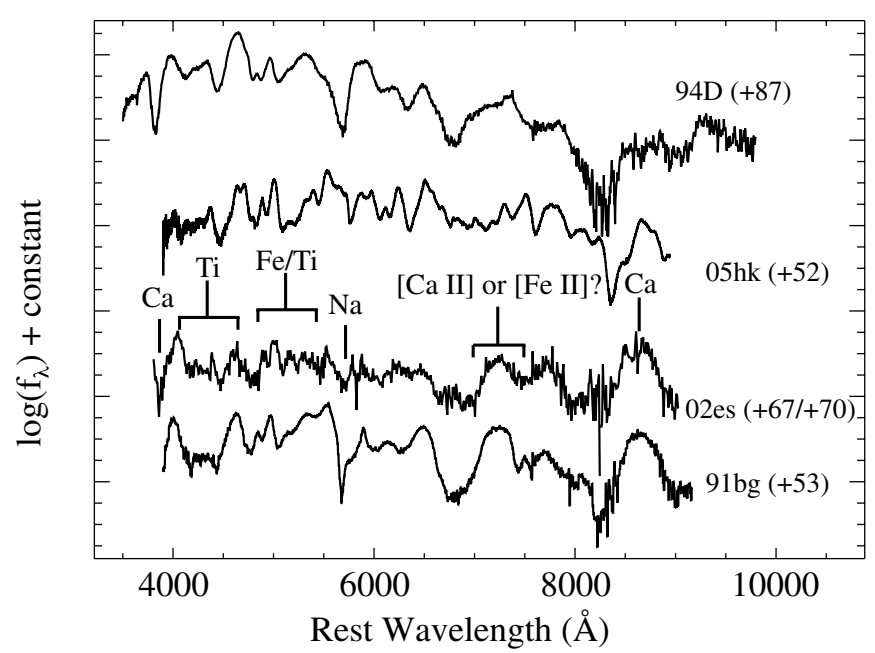

Figure 9. Combined spectrum of SN 2002es from +67 days and +70 days after maximum light. For comparison we show the normal SN 1994D (from our database of spectra), SN 2005hk (Phillips et al. 2007), and SN 1991bg (Turatto et al. 1996) at a similar phase indicated in parentheses to the right of each spectrum. All spectra have been corrected for both Milky Way and hostgalaxy extinction using the values in their respective references. At this phase, SN 2002es looks similar to SN 1991bg, but with narrower features. Major spectroscopic features are identified.

\subsection{SYNOW Modeling}

The supernova spectral synthesis code SYNOW (Fisher et al. 1997 ) is useful in identifying the different ion contributions in SN spectra. We use SYNOW as a tool to analyze our +3 day, +13 day, and +37 day spectra to determine the elemental composition of the photosphere. Our synthetic spectra compared to our observed spectra, along with identification of major spectral features, can be found in Figure 10.

For our +3 day spectrum, we set the blackbody temperature to $13,000 \mathrm{~K}$ with a photospheric velocity of $6200 \mathrm{~km} \mathrm{~s}^{-1}$. Many of the ions present in our SYNOW spectrum are commonly found in the spectra of SN 1991bg-like objects: Ca II, Ti II, O I, Si II, S II, and Na I. Sullivan et al. (2011b) find evidence of Sc II in spectra of PTF 09dav, a peculiar subluminous SN Ia similar in some characteristics to SN 2002es. We find that including Sc II does not improve the fit to the spectrum of SN 2002es.

Just redward of Si II $\lambda 6355$ is a notch that looks suspiciously like $\mathrm{C}_{\text {II }} \lambda 6580$. The presence of carbon in pre-maximum spectra has recently been found in a substantial fraction $(\sim 20 \%-30 \%)$ of SNe Ia (Parrent et al. 2011; Thomas et al. 2011; Folatelli et al. 2012; Silverman \& Filippenko 2012) at velocities slightly higher than those of intermediate-mass elements such as $\mathrm{Si}$ and $\mathrm{Ca}$. If the notch is due solely to $\mathrm{C}_{\mathrm{II}}$, it has a velocity of $4200 \mathrm{~km} \mathrm{~s}^{-1}$, much lower than the velocities of other ions. With SYNOW, we can fit this feature reasonably well with a blend of C II and Co II with a velocity of $6200 \mathrm{~km} \mathrm{~s}^{-1}$. We do not see evidence of $\mathrm{C}_{\text {II }} \lambda 7234$. The notch is also present in our spectrum at +13 days, but it is not generally visible in normal SNe Ia that exhibit carbon at early times before maximum light. Persistent carbon features lasting two weeks were seen in the exceptionally luminous SN 2009dc (Taubenberger et al. 2011). However, given the subluminous nature of SN 2002es, it shares few characteristics in common with SN 2009dc (Yamanaka et al. 2009; Silverman et al. 2011; Taubenberger et al. 2011) and similar overly luminous SNe Ia (Howell 2001; Hicken et al. 2007; Scalzo et al. 2010). 


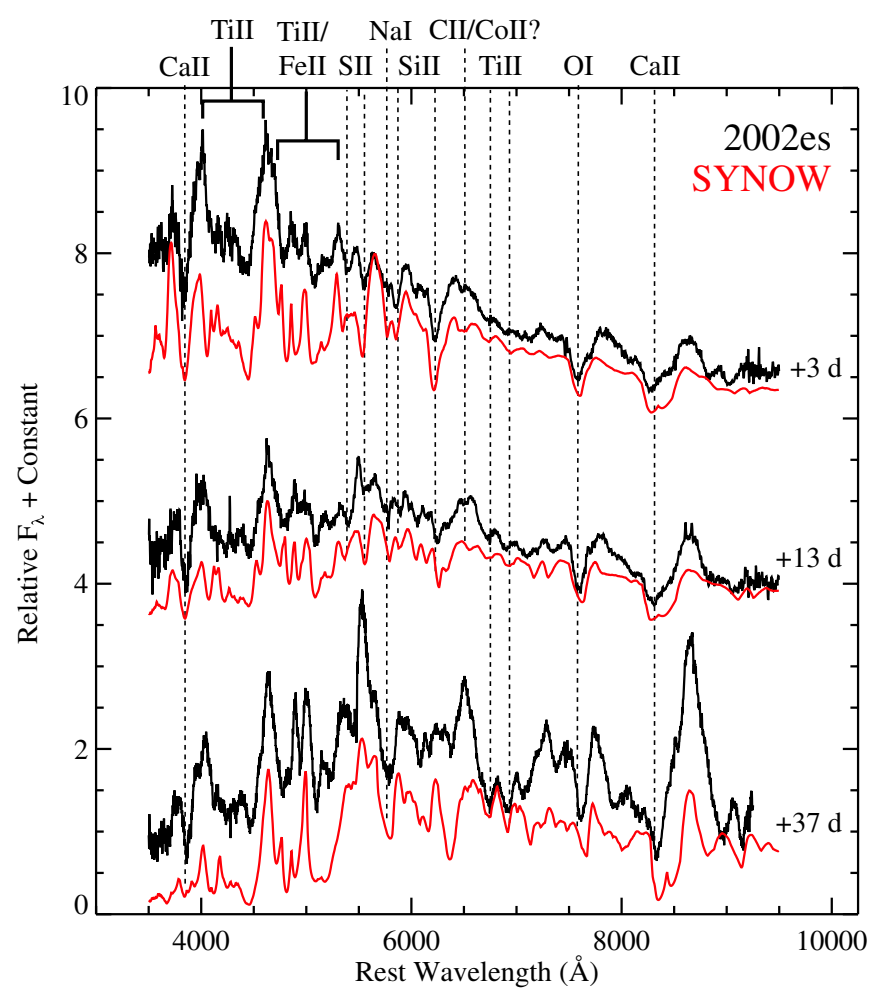

Figure 10. Spectra of SN 2002es at +3 days, +13 days, and +37 days after maximum light compared to our best SYNOW fit for each epoch in red. Ions that contribute to major features are labeled according to the blueshifted absorption minimum of that feature. Our tentative identification of $\mathrm{C} \mathrm{II}$ is indicated by a question mark.

(A color version of this figure is available in the online journal.)

In an analysis of the pre-maximum spectrum of SN 2006bt, Foley et al. (2010b) found possible evidence for C $\mathrm{II}$ at lower velocities $\left(\sim 5200 \mathrm{~km} \mathrm{~s}^{-1}\right)$ than other ions $\left(\sim 12,500 \mathrm{~km} \mathrm{~s}^{-1}\right)$. However, unlike the case in SN 2002es, they saw that the feature disappeared after maximum light. This feature, whether attributed to $\mathrm{C}$ II or not, hints at a possible connection between the two transients.

In our +13 day spectrum, we set the blackbody temperature to $7500 \mathrm{~K}$ with a photospheric velocity of $5500 \mathrm{~km} \mathrm{~s}^{-1}$. The low velocities of the ions allow for the detection of a forest of absorption lines normally smeared out by higher velocities. We see less evidence for the presence of Si II and S II. Absorption from iron-group elements such as Fe II and Ti II becomes more prominent.

By +37 days, we use a blackbody temperature of $5900 \mathrm{~K}$ with a photospheric velocity of $3500 \mathrm{~km} \mathrm{~s}^{-1}$. At this phase, we begin to see that the SN become nebular and there is significant line blending at short wavelengths from iron-group elements. We still detect continuum emission, indicating that the $\mathrm{SN}$ is not completely nebular, but we begin to see emission from calcium.

\subsection{Quantitative Measurements}

SNe Ia can be broadly classified by a clustering analysis of spectral and photometric features (Benetti et al. 2005; Branch et al. 2006). Most quantitative measurements make use of Si II features at $5972 \AA$ and Si II $\lambda 6355$. Nugent et al. (1995) introduced $\mathcal{R}(\mathrm{Si})$, the ratio of the depth of $\mathrm{Si}$ II $\lambda 5972$ to $\lambda 6355$ near maximum light, which is found to correlate with light-curve width. Using $\mathcal{R}(\mathrm{Si})$ and the velocity gradient of the $\mathrm{Si}$ II $\lambda 6355$

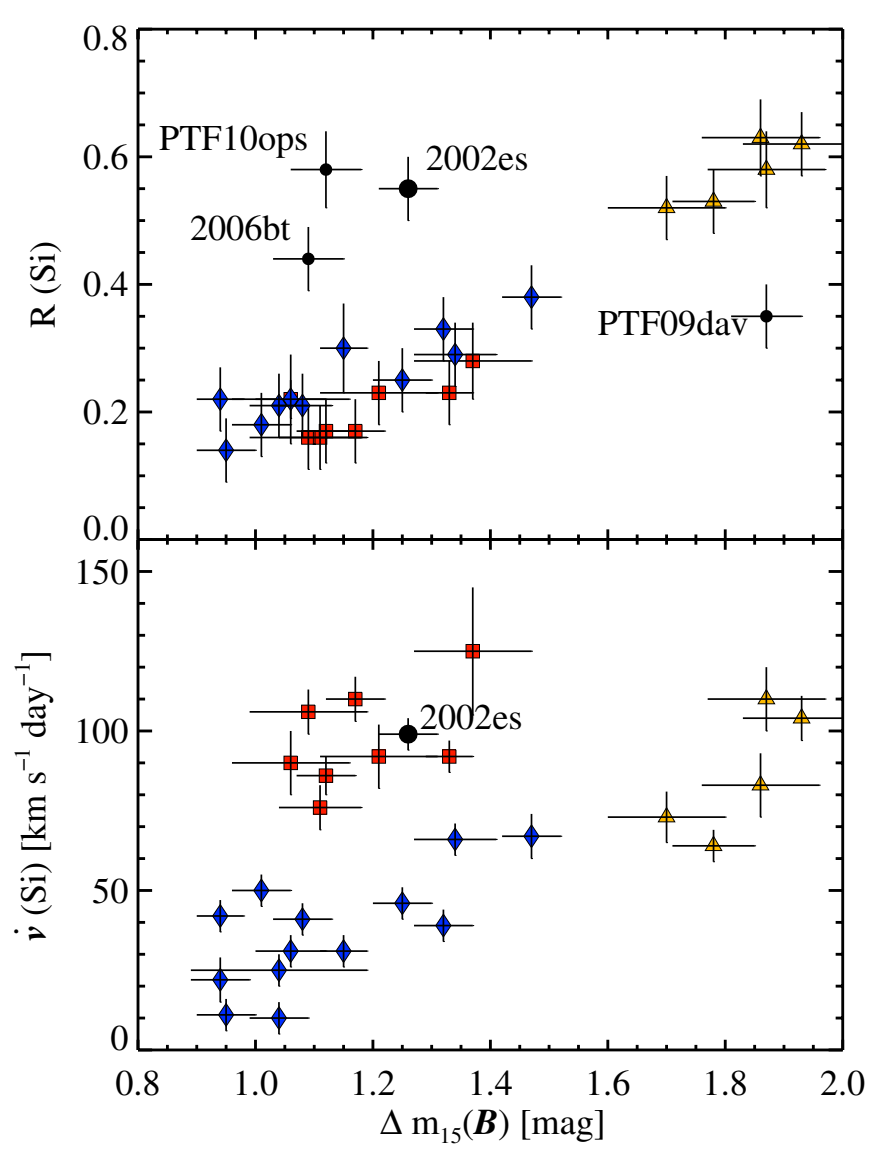

Figure 11. Top panel: the silicon ratio near maximum light, $\mathcal{R}$ (Si II), vs. $\Delta m_{15}(B)$. Blue diamonds are low-velocity objects, red squares are high-velocity objects, and yellow triangles are FAINT objects as defined by Benetti et al. (2005). SN 2002es joins PTF 10ops and SN 2006bt as outliers in the relationship between $\mathcal{R}$ (Si II) and $\Delta m_{15}(B)$. SN 2002es has an $\mathcal{R}(\mathrm{Si}$ II) more typical of a FAINT object, despite having a broad light curve. Bottom panel: gradient in the velocity of the Si II feature vs. $\Delta m_{15}(B)$. Based on the clustering of this plot, SN 2002es would be classified as a HVG object despite being subluminous.

(A color version of this figure is available in the online journal.)

feature, $\dot{v}$, Benetti et al. (2005) found that SNe Ia could be broken into three distinct subclasses: low velocity gradient, high velocity gradient (HVG), and FAINT.

We estimate $\mathcal{R}(\mathrm{Si})=0.55 \pm 0.05$ from our earliest spectrum of SN 2002es taken +3 days after maximum light. In the top panel of Figure 11, we plot $\Delta m_{15}(B)$ against $\mathcal{R}(\mathrm{Si})$ for the objects in Benetti et al. (2005) along with SN 2002es and other peculiar subluminous objects. While SN 2002es has an $\mathcal{R}(\mathrm{Si})$ value similar to that of FAINT objects, its measured $\Delta m_{15}(B)$ does not match those of FAINT objects. Compared to other peculiar subluminous SNe Ia, SN 2002es is most similar to PTF 10ops (Maguire et al. 2011), both of which are significant outliers in the relationship between $\Delta m_{15}(B)$ and $\mathcal{R}(\mathrm{Si})$ found by Benetti et al. (2005).

We measure $\dot{v}=98 \pm 5 \mathrm{~km} \mathrm{~s}^{-1}$ day $^{-1}$ (statistical error only) from six spectra taken in the range $+3 \leqslant t \leqslant+13$ days. In the bottom panel of Figure 11, we plot $\Delta m_{15}(B)$ against $\dot{v}$ for SN 2002es and the objects in Benetti et al. (2005), coded by subclass. Based on our measured $\dot{v}$ and $\Delta m_{15}(B), \mathrm{SN} 2002 \mathrm{es}$ falls into the HVG group. FAINT objects also have HVGs, but they have a larger $\Delta m_{15}(B)$ as well (i.e., a narrow light curve). SN 2002es would fall into the FAINT classification if it had a narrower light curve. Both panels indicate that SN 2002es has a 
unique combination of photometric and spectroscopic properties that lie outside the classification scheme of Benetti et al. (2005).

\section{DISCUSSION}

\subsection{Bolometric Luminosity}

Estimating the bolometric luminosity is particularly difficult without observations in the ultraviolet (UV) and IR, which contribute a sizable fraction of energy for normal SNe Ia. Unfortunately, $U$-band photometry from Hicken et al. (2009a) starts $\sim 5$ days after $B$-band maximum light. We only make use of $B V R I$ data to construct a "quasi-bolometric" (Nomoto et al. 1990) luminosity light curve for SN 2002es.

We estimate the quasi-bolometric luminosity by warping spectra representing the SED of SN 2002es corrected for Milky Way extinction to match our multi-color photometry using a method similar to that of Howell et al. (2009). We assume there is no host-galaxy extinction (see Section 2.3). For each epoch of $B V R I$ photometry, we warp the spectrum using a third-order spline with knots at the effective wavelength of each filter to match the photometric colors. In instances where we are missing photometry from one band, we do a linear interpolation between the nearest photometric epochs. We note that there is a fairly large gap in our $B$-band photometry at $+40<t<+70$ days, making our interpolation uncertain. The resulting warped spectrum is integrated over the range 4000-8800 $\AA$ (i.e., from the blue limit of the $B$ band to the red limit of the $I$ band) to obtain the optical flux for the photometric epoch. The flux is converted to a luminosity using the distance reported in Section 2.3.

Ideally, a spectral series of SN 2002es matched with each photometric epoch (as opposed to a single spectrum) should be used for the most accurate results. The spectra from FAST presented in this paper do not extend to the $I$ band, and thus are not useful for estimating the bolometric luminosity. Given the spectroscopic similarities between SN 1991bg and SN 2002es, we used the above procedure with the SN 1991bg spectral series of Nugent et al. (2002) artificially redshifted by 0.01 to match the slower expansion velocities of SN 2002es.

A bolometric correction is required to turn our quasibolometric luminosity based on optical data into a bolometric luminosity that accounts for energy emitted in the UV and IR. Our SN 1991bg spectral series covers 1000-25,000 ̊ and provides a reasonable first-order approximation of the SED of SN 2002es. We calculate the fraction of flux emitted in the range 4000-8800 $\AA$ compared to the total integrated flux for each spectrum in the series. This provides us with an estimate of the flux missed by only using optical data to construct our quasi-bolometric light curve as a function of phase. We apply this correction to arrive at our final bolometric light curve. At maximum light, the BVRI data account for $\sim 70 \%$ of the total flux. Given the uncertainties in our estimates, we include a $20 \%$ systematic error in our error budget for the bolometric luminosity.

We estimate $L_{\text {bol }}=(4.0 \pm 0.9) \times 10^{42} \mathrm{erg} \mathrm{s}^{-1}$ at $\sim 1$ day after maximum light in the $B$ band. This is slightly larger than the bolometric luminosity of other subluminous SNe Ia (Taubenberger et al. 2008) and $\sim 25 \%$ of the luminosity of SN 2005cf (Wang et al. 2009b).

The luminosity of thermonuclear $\mathrm{SNe}$ is powered by the energy deposition of $\gamma$-rays and positions produced by the radioactive decay chain ${ }^{56} \mathrm{Ni} \rightarrow{ }^{56} \mathrm{Co} \rightarrow{ }^{56} \mathrm{Fe}$. At maximum light, the rate of energy deposition into the expanding ejecta is roughly equivalent to the luminosity of the SN (i.e., Arnett's law; Arnett 1982). Following Stritzinger \& Leibundgut (2005), we can write Arnett's law as

$$
\begin{aligned}
L_{\mathrm{bol}}=\alpha & \times\left(6.45 e^{-t_{r} /(8.8 \text { days })}+1.45 e^{-t_{r} /(111.3 \text { days })}\right) \\
& \times\left(\frac{M_{\mathrm{Ni}}}{M_{\odot}}\right) \times 10^{43} \mathrm{erg} \mathrm{s}^{-1},
\end{aligned}
$$

where $\alpha$ is a correction factor of the order of unity to Arnett's law and $t_{r}$ is the time between explosion and maximum light (i.e., the bolometric rise time).

Assuming that the luminosity of SN 2002es is powered by the decay of ${ }^{56} \mathrm{Ni}$, we can estimate the amount of ${ }^{56} \mathrm{Ni}$ synthesized in the explosion. We set $\alpha=1$. Unfortunately, we do not have tight constraints on the date of explosion. Prior to the first detection of SN 2002es on 2002 August 23.2 ( $\sim 9$ days before the time of maximum $L_{\mathrm{bol}}$ ), KAIT obtained an unfiltered image of the field on 2002 August 12.5 with a limiting magnitude of 18.5 (Li et al. 2002). Ganeshalingam et al. (2011) found that the subluminous SN 1999by had a rise time to maximum light in $B$ of $13.33 \pm 0.40$ days, consistent with other estimates for the rise time of SN 1991bg-like SNe from Taubenberger et al. (2008), while a typical, normal SN Ia has a rise time of $\sim 18$ days. The $B$-band light curve of SN 2002es has a slower rise to maximum light than SN 1999by and matches the rise of SN 2005hk. Phillips et al. (2007) measure a rise to $B$-band maximum of $15 \pm 1$ days for the SN 2002cx-like SN 2005hk. Maguire et al. (2011) constrain the rise time of PTF 10ops, an object that shares similarities to SN 2002es, to $\sim 19$ days. Based on the unique spectroscopic and photometric peculiarities of SN 2002es, it is unclear which object serves as the best guide to determining the rise time. As a compromise between the different possible rise times, we adopt a rise time to bolometric maximum of $t_{r}=16 \pm 3$ days.

From Equation (1), we estimate $M_{\mathrm{Ni}}=0.17 \pm 0.05 M_{\odot}$ synthesized in the explosion, which falls at the low end of the range $0.05<M_{\mathrm{Ni}}<0.87 M_{\odot}$ found by Stritzinger et al. (2006b) for a sample of SNe Ia. SN 1991bg and SN 1999by synthesized $\sim 0.1 M_{\odot}$ of ${ }^{56} \mathrm{Ni}$.

\subsection{Energetics}

We can estimate the ejected mass, $M_{\mathrm{ej}}$, and the kinetic energy, $E_{0}$, of the explosion using the rise time. Following the treatments of Arnett (1982) and Pinto \& Eastman (2000a, 2000b), we have $t_{r}^{2} \propto \kappa M_{\mathrm{ej}} / v_{s}$, where $\kappa$ is the mean opacity and $v_{s}$ is the ejecta velocity. If we compare SN 2002es to a normal SN Ia with $M_{\mathrm{ej}} \approx 1.4 M_{\odot}, v_{s}=10^{4} \mathrm{~km} \mathrm{~s}^{-1}$, and $t_{r}=18$ days, and assume that they have similar opacities, we have

$$
M_{\mathrm{ej}}=0.66 M_{\odot}\left(\frac{t_{r}}{16 \text { days }}\right)^{2}\left(\frac{v_{s}}{6000 \mathrm{~km} \mathrm{~s}^{-1}}\right) .
$$

The largest uncertainty is our calculation is from our estimate of the rise time of $t_{r}=16 \pm 3$ days. Including this uncertainty, we find $M_{\mathrm{ej}}=0.66 \pm 0.25$ for our nominal values, significantly lower than the canonical values for an SN Ia.

We can then calculate the kinetic energy

$$
E_{\mathrm{k}}=2.4 \times 10^{50} \mathrm{erg}\left(\frac{t_{r}}{16 \text { days }}\right)^{2}\left(\frac{v_{s}}{6000 \mathrm{~km} \mathrm{~s}^{-1}}\right)^{3}
$$

Including the uncertainty in our rise time gives $E_{\mathrm{k}}=(2.4 \pm$ $0.9) \times 10^{50}$ erg for our nominal values. 
The estimated ejected mass and kinetic energy are significantly lower than the canonical values for an SN Ia in part due to the slow expansion velocities measured from the $\mathrm{Si}$ feature. We again caution that our estimate for the rise time is not well constrained and can range from 13 days to 19 days.

Following similar arguments made by Howell et al. (2006) and Silverman et al. (2011), we can place constraints on the WD progenitor mass based on the energetics of the explosion. We construct a series of WD models with central temperature $T=10^{7} \mathrm{~K}$ using the Models for Experiments in Stellar Astrophysics (MESA) code (Paxton et al. 2011) and calculate the binding energy for each model. We find that the nuclear energy released is larger than the combined binding energy and kinetic energy for all of our models. This ensures that there is enough energy to unbind the WD and power the explosion based on the ${ }^{56} \mathrm{Ni}$ mass, but it is unclear what happens to the excess nuclear energy. Realistically, some of the nuclear energy should go into heating the ejecta. If some fraction of the WD was left unburned, then nuclear energy would decrease. However, our evidence for unburned carbon (see Section 3.4) is questionable. Given the uncertainty in our assumptions, we cannot place a strong constraint on the mass of the progenitor.

\subsection{Late-time Decay}

We build a simple toy model to describe the luminosity due to the deposition of energy from the radioactive decay of ${ }^{56} \mathrm{Ni}$ and ${ }^{56} \mathrm{Co}$ to study the behavior of the bolometric light curve. The energy initially deposited into the ejecta is from the thermalization of $\gamma$-rays emitted by radioactive decay of ${ }^{56} \mathrm{Ni} \rightarrow{ }^{56} \mathrm{Co}$ with an $e$-folding time of 8.8 days. By $\sim 20$ days, the energy emitted by the decay of ${ }^{56} \mathrm{Co} \rightarrow{ }^{56} \mathrm{Fe}$ exceeds that of ${ }^{56} \mathrm{Ni}$ ( $e$-folding time of 111.3 days). The decay of ${ }^{56} \mathrm{Co}$ can proceed via either electron capture emitting a spectrum of $\gamma$-rays $(96.5 \%$ of emitted energy) or beta decay releasing a positron (3.5\% of emitted energy) (Nadyozhin 1994). The luminosity due to the radioactive decay can be written as

$$
\begin{aligned}
L_{\mathrm{rad}}= & L_{\mathrm{Ni}, \gamma}\left[1-e^{-\tau}\right]+L_{\mathrm{Co}, \mathrm{e}^{+}} \\
& +L_{\mathrm{Co}, \gamma}\left[1-e^{-\tau}\right],
\end{aligned}
$$

where the factor $\left(1-e^{-\tau}\right)$ is the fraction of $\gamma$-rays trapped in the expanding ejecta with an optical depth $\tau$. We assume that the positrons are fully trapped within the ejecta and deposit their kinetic energy instantaneously. Note that $\tau$ is a function of time, decreasing as the ejecta expand homologously. Following Sollerman et al. (2002, 2004) and Stritzinger et al. (2006a), we write the optical depth to $\gamma$-rays in expanding ejecta as

$$
\tau=\left(\frac{t_{0}}{t}\right)^{2},
$$

where $t_{0}$ is the fiducial time relative to explosion when the ejecta become optically thin to $\gamma$-rays.

Solving the first-order differential equations that describe the parent-daughter relationship between ${ }^{56} \mathrm{Ni}$ and ${ }^{56} \mathrm{Co}$, we write the radioactive luminosity as

$$
\begin{aligned}
L_{\mathrm{rad}}= & \lambda_{\mathrm{Ni}} Q_{\mathrm{Ni}, \gamma} N_{\mathrm{Ni}, 0} e^{-\lambda_{\mathrm{Ni}} t}\left[1-e^{-\tau}\right] \\
& +\frac{\lambda_{\mathrm{Ni}} \lambda_{C o}}{\lambda_{\mathrm{Ni}}-\lambda_{\mathrm{Co}}} N_{\mathrm{Ni}, 0}\left(e^{-\lambda_{\mathrm{Co}} t}-e^{-\lambda_{\mathrm{Ni}} t}\right) \\
& \times\left[Q_{\mathrm{Co}, \mathrm{e}^{+}}+Q_{\mathrm{Co}, \gamma}\left(1-e^{-\tau}\right)\right],
\end{aligned}
$$

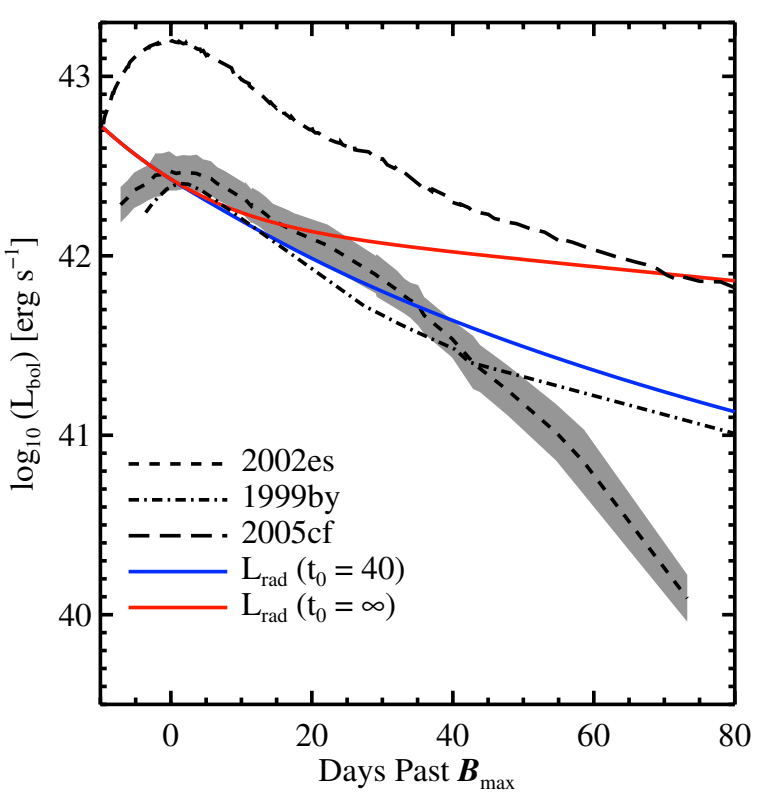

Figure 12. Bolometric luminosity of SN 2002es (dashed line) constructed using $B V R I$ data and bolometric corrections from SN 1991bg. The shaded region indicates the $1 \sigma$ errors. Plotted for comparison is the bolometric luminosity of SN 2005cf (long-dashed line; Wang et al. 2009b) and SN 1999by (dot-dash line; Garnavich et al. 2004). The solid blue and dotted red curves are models for the radioactive luminosity using Equation (6) assuming different $\gamma$-ray trapping efficiencies with an initial $M_{\mathrm{Ni}}=0.17 M_{\odot}$. The red dotted curve assumes full trapping of $\gamma$-rays producing a late-time slope that follows the cobalt decay rate. The blue curve is our best fit. Neither curve can reproduce the steep late-time decay in the bolometric light curve of SN 2002es.

(A color version of this figure is available in the online journal.)

where $\lambda_{\mathrm{Ni}}$ and $\lambda_{\mathrm{Co}}$ are the inverse $e$-folding times for ${ }^{56} \mathrm{Ni}$ and ${ }^{56} \mathrm{Co}$, respectively; $N_{\mathrm{Ni}, 0}$ is the initial amount of ${ }^{56} \mathrm{Ni}$ synthesized in the explosion; $Q_{\mathrm{Ni}, \gamma}(1.75 \mathrm{MeV})$ is the energy yielded by each ${ }^{56} \mathrm{Ni} \rightarrow{ }^{56} \mathrm{Co}$ decay; and $Q_{\mathrm{Co}, \gamma}(3.61 \mathrm{MeV})$ and $Q_{\mathrm{Co}, \mathrm{e}^{+}}(0.12 \mathrm{MeV})$ are the energy yielded per ${ }^{56} \mathrm{Co} \rightarrow{ }^{56} \mathrm{Fe}$ decay via electron capture and beta decay, respectively.

In Figure 12, we plot the bolometric light curve of SN 2002es (see Section 4.1 for details), as well as the UV-optical-IR (UVOIR) bolometric light curves of SN 2005cf from Wang et al. (2009b) and SN 1999by using data from Garnavich et al. (2004). We also plot models $L_{\mathrm{rad}}\left(t_{0}=40\right.$ days) and $L_{\mathrm{rad}}\left(t_{0}=\right.$ $\infty$ days) assuming $M_{\mathrm{Ni}}=0.17 M_{\odot}$ (found using Arnett's law in Section 4.1). The model with $t_{0}=\infty$ represents the case in which the $\gamma$-rays are completely trapped and the luminosity decays according to the ${ }^{56} \mathrm{Co}$ decay rate $\left(0.01 \mathrm{mag} \mathrm{day}^{-1}\right)$.

The bolometric light curve of SN 2002es shows a surprising drop in luminosity at $t>+30$ days. The decline is significantly faster than the cobalt decay rate and the decline rate for SN 2005cf. Similar results were found for the late-time decline in individual optical bands (see Section 3.1). Even when accounting for a $\gamma$-ray optical depth that decreases with time due to homologous expansion of the ejecta, we are unable to find a model that reasonably matches the bolometric light-curve decline rate of SN 2002es. Our best match is $t_{0}=40$ days, which adequately describes SN 2002es within the region $0<$ $t<+30$ days but fades too slowly at $t>+30$ days.

The decay of the bolometric light curve in the range $+30<$ $t<+70$ days appears incompatible with the gradual escape of $\gamma$-rays through homologously expanding ejecta and implies that the ejecta become optically thin to $\gamma$-rays very rapidly. If the ejecta are optically thin to $\gamma$-rays, then the luminosity should 
be powered by the thermalized kinetic energy of positrons, assuming some fraction of the positrons are trapped in the ejecta. We estimate the nickel mass required to power the light curve by positrons at our last photometry epoch $(t=89.3 \pm 3$ days after explosion) using Equation (6) and setting $\tau=0$ (i.e., the ejecta are optically thin to $\gamma$-rays). For complete positron trapping, we estimate that $M_{\mathrm{Ni}}=0.05 \pm 0.02 M_{\odot}$ is required to power the luminosity at this phase; this is a factor of three less than our previous estimate using Arnett's law at maximum light. A corollary to the assumption of complete positron trapping is that the light-curve decay should follow the cobalt decay rate, which is clearly not seen. If we allow for only partial trapping, we need a positron trapping fraction of $\sim 0.3$ to match our $M_{\mathrm{Ni}}$ from Arnett's law.

The unexpectedly fast decline calls into question whether SN 2002es was powered by ${ }^{56} \mathrm{Ni}$ decay. Here, we discuss possible ways to reconcile a thermonuclear $\mathrm{SN}$ with the measured decline rate.

Dust formation around the SN would lead to a drop in optical flux as high-energy photons are reprocessed to longer IR wavelengths. In models of dust formation in the ejecta of SNe Ia, Nozawa et al. (2011) find that the conditions required to produce dust occur 100-300 days after maximum light. However, due to the low densities in SN Ia ejecta, the dust grains are small $(<0.01 \mu \mathrm{m})$, and the expected IR emission associated with dust has not yet been detected in late-time observations of SNe Ia (Gerardy et al. 2007). We also do not find evidence of an increase in the red continuum in our spectra or asymmetries in line features in our late-time spectra as have been seen in other SNe that formed dust (e.g., Smith et al. 2008). Our last epoch of photometry taken +73 days after maximum light gives $B-R \approx 0.5 \mathrm{mag}$, while photometry of SN 2005cf taken from Wang et al. (2009b) gives $B-R \approx 0.7$ mag. SN 2002es is bluer than SN 2005cf, which is opposite of the effect we would expect if dust were facilitating the rapid fading.

Axelrod (1980) predicts that SNe Ia should undergo an "infrared catastrophe" (IRC) at late times, once the temperature drops below a critical threshold $(\sim 1000 \mathrm{~K})$. Models of the IRC predict that a thermal instability shifts the bulk of emission from the optical to fine-structure transitions of iron in the IR about 500-700 days after maximum light. Without IR data, we are unable to determine whether the IR flux increases as the optical flux decreases, although the onset of the IRC at such an early phase is certainly unexpected. Our last spectrum of SN 2002es taken 70 days after maximum light shows permitted lines in absorption, indicating that the ejecta have not yet become nebular and the temperature has probably not dropped sufficiently low to facilitate the onset of the IRC. The IRC has never been detected in late-time observations of other $\mathrm{SNe}$ Ia, although Leloudas et al. (2009) evoke the possibility of the IRC occurring locally in clumpy ejecta to explain the missing flux in the late-time light curve of SN $2003 \mathrm{hv}$.

\subsection{A Pure Explosion Model}

Another possible model for the evolution of the light curve of SN 2002es is that the energy deposited into the ejecta is derived from the explosion of the progenitor, and there is no subsequent heating. Such a model was explored by Kasliwal et al. (2010) to explain the rapidly evolving SN 2010X. The explosion energy $\left(E_{0}\right)$ is deposited instantaneously into the ejecta. At maximum light, $L_{\max } \approx E_{0} / t_{d}(0)$, where $t_{d}(0)$ is the initial diffusion time for photons through the ejecta. The diffusion time is $t_{d}(0) \propto M_{\mathrm{ej}} \kappa / R_{0}$, where $R_{0}$ is the initial radius of the $\mathrm{SN}$.
Assuming $\kappa=0.1 \mathrm{~cm}^{2} \mathrm{~g}^{-1}$ for Fe-rich ejecta (Pinto \& Eastman 2000b), $L_{\max }=4.0 \times 10^{42} \mathrm{erg} \mathrm{s}^{-1}$, and $M_{\mathrm{ej}}=0.66 M_{\odot}$, we estimate an initial progenitor radius of $R_{0} \approx 10^{12} \mathrm{~cm}$.

This value is similar to the estimate presented by Kasliwal et al. (2010), in which they argue that such a radius would require a progenitor with an extended hydrogen envelope. Based on the absence of hydrogen in spectra of SN 2010X they reject this hypothesis. Similarly, given the lack of hydrogen in our spectra of SN 2002es, we also find a pure explosion an unlikely mechanism to power the luminosity of SN 2002es.

\subsection{A Core-collapse SN?}

If SN 2002es was not powered by ${ }^{56} \mathrm{Ni}$ decay, it is reasonable to investigate core-collapse mechanisms that could explain the properties of SN 2002es. However, the lack of hydrogen emission in optical spectra of SN 2002es rules out a massive star with a large hydrogen envelope as a progenitor, leaving the possibility that SN 2002es could be an SN Ic. The star formation history of the host galaxy strongly favors an older stellar population, making the progenitors of SNe Ibc unlikely as well (Leaman et al. 2011). If SN 2002es is a core-collapse event, it would have to be the result of a low-mass star following an atypical evolutionary path to an SN Ic.

Perets et al. (2010) discussed data on SN 2005E, a lowluminosity SN Ib (based on the absence of hydrogen and the presence of helium) with calcium-rich ejecta that exploded in the outskirts of its early-type host galaxy. Although the observed properties of SN 2005E and SN 2002es are different (e.g., SN 2002es is much brighter, has slower expansion velocities, and no detectable helium), SN 2005E and other objects like it may be core-collapse SNe connected to old stellar populations. Kasliwal et al. (2011) studied the observed properties of the emerging class of Ca-rich objects and found that they could not be explained by conventional core-collapse or thermonuclear explosions.

Another possible atypical core-collapse object was SN 2008ha, a low-luminosity Type I SN (based on the absence of hydrogen) that peaked at -14.2 mag with extremely low ejecta velocities ( 4000-5000 $\mathrm{km} \mathrm{s}^{-1}$; Valenti et al. 2009; Foley et al. 2009, 2010a). Valenti et al. (2009) argued that SN 2008ha and the family of SN 2002cx-like objects may be the result of the core collapse of hydrogen-poor, low-mass stars. Their analysis of SN 2008ha spectra showed little evidence for the IMEs commonly associated with the byproducts of a thermonuclear explosion and an absence of forbidden iron lines at late times. Coupled with the low luminosity and low ejecta velocities, the authors interpret SN 2008ha as a core-collapse event that produced little ${ }^{56} \mathrm{Ni}$. However, Foley et al. (2010a) presented an early-time spectrum of SN 2008ha that clearly exhibits IMEs such as silicon, sulfur, and carbon, arguing in favor of the thermonuclear explosion of a $\mathrm{C} / \mathrm{O} \mathrm{WD}$.

\subsection{SN 1999bh: An SN 2002es-like Object}

Combing through our photometric (Ganeshalingam et al. 2010) and spectral (Silverman et al. 2012b) databases, we recognized that $\mathrm{SN} 1999 \mathrm{bh}$ shares many of the same properties as SN 2002es. Although our photometric and spectroscopic coverage of SN 1999bh is not as extensive as our SN 2002es data set, the available data provide a compelling case to link the two objects.

BVRI photometry of $\mathrm{SN} 1999 \mathrm{bh}$ are taken from Ganeshalingam et al. (2010). Spectra of the object were 
Table 5

Log of Optical Spectral Observations of SN 1999bh

\begin{tabular}{lccccc}
\hline \hline UT Date & $\begin{array}{c}\text { Phase }^{\mathrm{a}} \\
(\text { days })\end{array}$ & Telescope/Instrument & $\begin{array}{c}\text { Exp. Time } \\
(\mathrm{s})\end{array}$ & $\begin{array}{c}\text { Res. }^{\mathrm{b}^{\mathrm{O}}} \\
(\AA)\end{array}$ & Observer $^{\mathrm{c}}$ \\
\hline 1999 Apr 09.3 & +6 & FLWO/FAST & 1200 & $6-7$ & MC \\
1999 Apr 24.4 & +21 & Lick/Kast & 1800 & $6-11$ & AF, WL \\
\hline
\end{tabular}

Notes.

a Rest-frame days relative to the date of $B_{\max }, 1999$ April 03.3 (JD 2,451,271.8), rounded to the nearest day.

b Approximate spectral resolution.

c AF: A. Filippenko; MC: M. Calkins; WL: W. Li.

obtained using the Kast dual spectrograph on the Shane $3 \mathrm{~m}$ telescope at Lick Observatory and the FAST spectrograph mounted on FLWO at Mount Hopkins. A journal of our observations is available in Table 5. The spectra were reduced using the techniques described in Section 2.2.

SN 1999bh was discovered in NGC 3435 by Li (1999) as part of LOSS on 1999 March 29.2. Subsequent spectroscopic follow-up observations by Aldering et al. (1999) classified the object as an SN Ia near maximum light on 1999 April 2. The authors estimate a redshift of 0.028 , likely based on SN features. The redshift of NGC 3435 listed in NED is $z_{\text {helio }}=0.0172$. We measure a redshift of $z_{\text {helio }}=0.0168$ from narrow $\mathrm{H} \alpha+[\mathrm{N}$ II] lines from the host galaxy in a spectrum of SN 1999bh taken on 1999 April 24 using the Kast dual spectrograph mounted on the Shane $3 \mathrm{~m}$ telescope. We adopt this redshift for the remainder of our analysis. The luminosity distance to NGC 3435 calculated using $z_{\mathrm{CMB}}=0.0172$ is $d_{L}=70.8 \pm 5.3 \mathrm{Mpc}$. At this redshift, $\mathrm{SN} 1999 \mathrm{bh}$ exploded at a projected distance of $3.5 \mathrm{kpc}$ from the nucleus of NGC 3435.

Figure 13 shows the BVRI light curves of SN 1999bh; we see that those of SN 2002es provide an excellent match in all bands. Both objects exhibit broad light curves despite being subluminous, and lack a prominent shoulder in the $R$ or $I$ bands.

Using SN 2002es as a template, we measure the date of maximum light to be JD 2, 451, 271.8 \pm 1 day (1999 April 3.3). After correcting for $E(B-V)=0.015$ mag from Milky Way extinction using the dust maps of Schlegel et al. (1998), we measure $B_{\max }=18.63 \pm 0.06 \mathrm{mag}$. Due to the faintness of the object, we were unable to follow SN 1999bh sufficiently long to measure the light-curve decay rates at $t>+30$ days.

Based on the presence of $\mathrm{Na}$ I D absorption at the redshift of NGC 3435 in spectra of SN 1999bh, we can be certain that there is some amount of extinction due to the host galaxy. We estimate an equivalent width (EW) of $0.8 \pm 0.2 \AA$ of $\mathrm{Na}$ I D absorption at the redshift of the host galaxy from our spectrum taken on 1999 April 9. Translating Na I D EW measurements to an inferred $E(B-V)$ reddening gives $0.1<E(B-V)<0.2$ mag depending on whether we use the relationship given by Barbon et al. (1990) or Turatto et al. (2003). Blondin et al. (2009) and Poznanski et al. (2011), however, have shown that while there is a positive correlation between the presence of $\mathrm{Na}$ I $\mathrm{D}$ absorption and host-galaxy extinction, $\mathrm{Na}$ I D absorption is not a strong predictor for the amount of extinction.

Instead, we estimate the amount of host-galaxy extinction by matching the $B-V$ color of $\mathrm{SN} 1999 \mathrm{bh}$ to that of SN 2002es. This assumes that any difference in colors is purely associated with host-galaxy extinction and not intrinsic differences between the colors of the two objects. We find that $E(B-V)_{\text {host }}=0.48 \pm 0.07 \mathrm{mag}$.

We also estimate the host-galaxy reddening by matching our SN 1999bh spectra to corresponding SN 2002es spectra. We

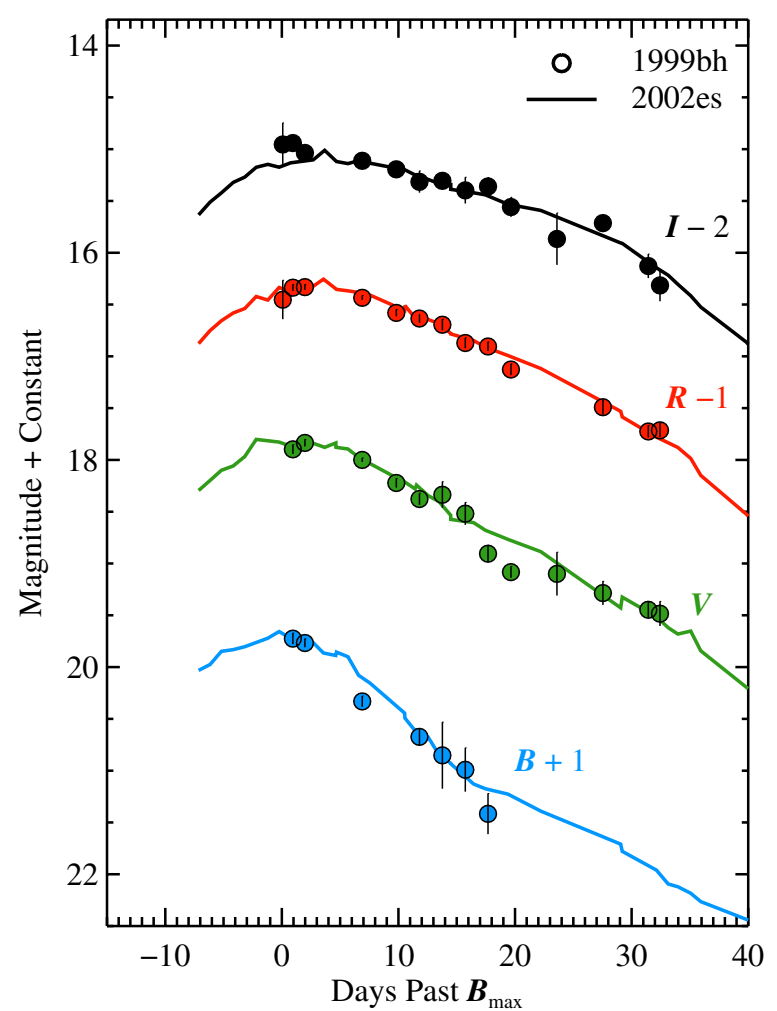

Figure 13. BVRI light curves of SN 1999bh (filled circles) in comparison to SN 2002es (solid lines). The light curves of both objects have been shifted relative to the time of $B_{\max }$ and peak magnitude. The light curves of SN 1999bh display a striking similarity to those of SN 2002es. Both sets of light curves are particularly broad, despite being subluminous and spectroscopically similar to those of SN 1991bg.

(A color version of this figure is available in the online journal.)

perform a fit to match the SED of the two objects, applying a CCM reddening law (Cardelli et al. 1989) with $R_{V}=3.1$ to deredden our SN 1999bh spectra. We obtain a best fit of $E(B-V)_{\text {host }}=0.59 \pm 0.01 \mathrm{mag}$ (statistical error only) matching our +5 day spectrum of SN 1999bh to our +3 day spectrum of SN 2002es, and $E(B-V)_{\text {host }}=0.49 \pm 0.01 \mathrm{mag}$ matching our +20 day spectrum of SN 1999 bh to our +13 day spectrum of SN 2002es. These values are consistent with what we derived above using the $B-V$ color at maximum light. We adopt $E(B-V)_{\text {host }}=0.48 \pm 0.07$ mag as the host-galaxy extinction.

In Figure 14, we show the color curves of SN 1999bh corrected for Milky Way and host-galaxy reddening. The curves of SN 2002es provide an excellent match for the color evolution of SN 1999bh. The $B-V$ and $V-R$ colors agree almost perfectly. The $V-I$ color of SN 1999 bh is bluer than expected, compared to that of SN 2002es. 


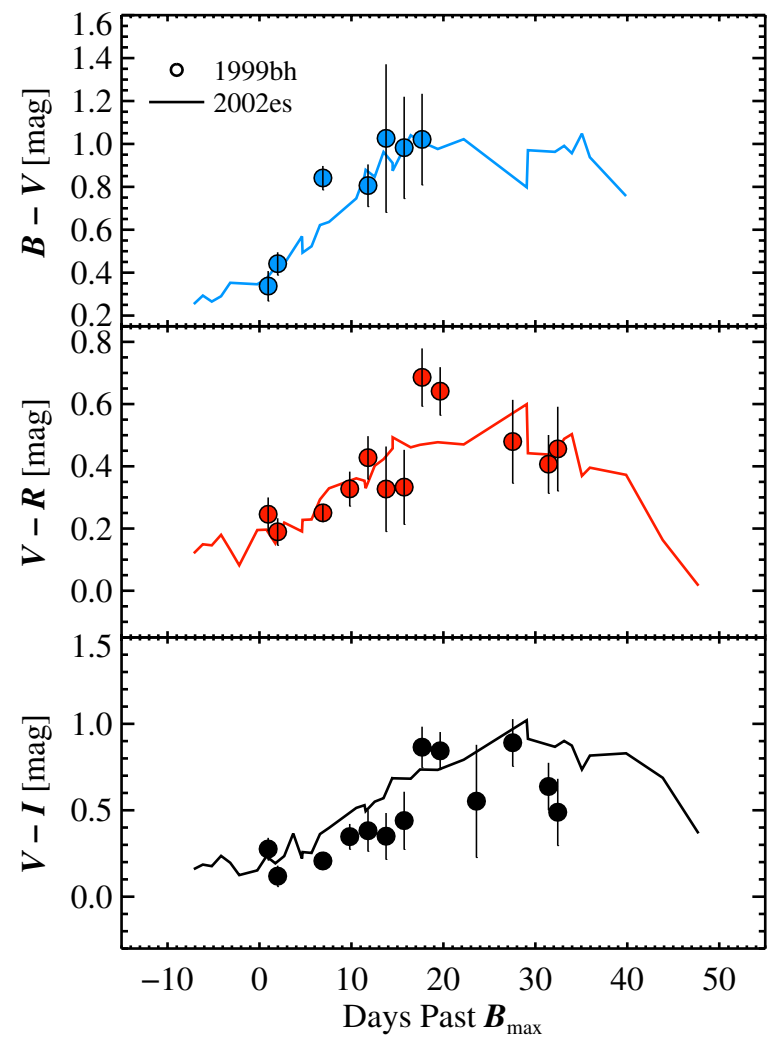

Figure 14. Color curves of SN 1999bh (solid circles) compared to SN 2002es (solid lines). SN 1999bh has been corrected for $E(B-V)_{\mathrm{MW}}=0.015 \mathrm{mag}$ and $E(B-V)_{\text {host }}=0.48 \mathrm{mag}$. The color curves of SN 2002es, corrected for $E(B-V)_{\mathrm{MW}}=0.183 \mathrm{mag}$, provide an excellent match for SN 1999bh.

(A color version of this figure is available in the online journal.)

After correcting for Milky Way and host-galaxy extinction, the absolute magnitude at peak of SN 1999bh was $M_{B}=$ $-17.71 \pm 0.27 \mathrm{mag}$, almost the same luminosity as SN 2002es. We estimate $\Delta m_{15}(B)=1.24 \pm 0.10$ mag. Having a light-curve width that is comparable to that of SN 2002es, SN 1999bh is another example of a $5 \sigma$ outlier in the Phillips relation as shown in Figure 3.

In Figure 15 we illustrate our two epochs of spectroscopy compared to SN 2002es at a comparable phase. In the top panel, we show our spectra around maximum light; SN 1999bh lacks hydrogen and exhibits $\mathrm{Si}$ II $\lambda 6355$, confirming its classification as an SN Ia. In addition, there is strong Ti II absorption, linking it to the SN 1991bg subclass. The ejecta velocity measured from the minimum blueshift of Si II $\lambda 6355$ is $\sim 6000 \mathrm{~km} \mathrm{~s}^{-1}$, similar to that of SN 2002es. In the bottom panel, we show our spectra from roughly three weeks after maximum light. Again, we see striking similarities between the two objects. We note that the narrow feature at the wavelength of $\mathrm{H} \alpha$ in both spectra is from the host galaxy.

The host of SN 1999bh is classified by van den Bergh et al. (2002) as an Sb galaxy. Given the narrow $\mathrm{H} \alpha$, [N II], and $\mathrm{Na}$ I D features superimposed on our spectra of SN 1999bh, it is likely that SN 1999bh lies along the line of sight of a star-forming region in NGC 3435. However, it is not possible to determine whether SN 1999bh is actually associated with the star-forming region or is behind it. Unlike the case for SN 2002es, the host galaxy of SN 1999bh does not help constrain the stellar population associated with SN 1999bh.

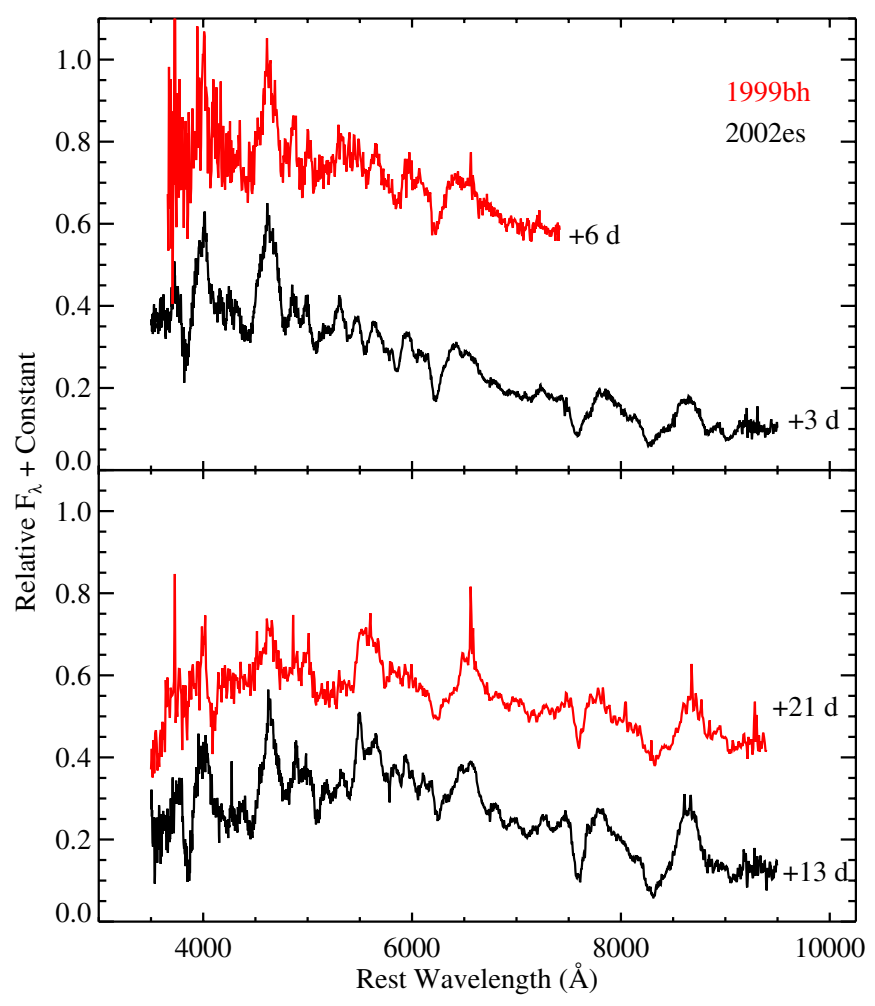

Figure 15. Spectra of SN 1999bh (red) in comparison to SN 2002es (black). The spectra have been corrected for the effects of host-galaxy recession. Spectra of SN 1999bh have been corrected for $E(B-V)=0.01$ mag due to Milky Way extinction and $E(B-V)=0.48$ mag from host-galaxy extinction using a CCM reddening law with $R_{V}=3.1$. Spectra of SN 2002es have been corrected for $E(B-V)=0.183$ mag due to Milky Way extinction.

(A color version of this figure is available in the online journal.)

\subsection{Rate}

Both SN 2002es and SN 1999bh were included in the LOSS SN rate study (Leaman et al. 2011; Li et al. 2011a, 2011b) in the luminosity function (LF) subsample used to calculate the volumetric rate for different SN Ia subtypes. The LF subsample is considered to represent a complete sample of $74 \mathrm{SNe}$ Ia within $80 \mathrm{Mpc}$. In Li et al. (2011b), both objects are classified as SN 2002cx-like SNe. Within a fixed volume, SN 2002es-like objects should account for $\sim 2.5 \%$ of SNe Ia. It is worth noting that the reclassification of SN 2002es and SN 1999bh decreases the reported volumetric fraction of SN 2002cx-like objects from $5.0 \%$ to $2.5 \%$ as well. We caution that our rate calculation is limited by the small number of SN 2002es-like objects in the LF sample.

\subsection{Comparison to Other Peculiar SN 1991bg-like SNe}

The spectroscopic subclass of SN 1991bg-like SNe has been shown to have their own form of Phillips relation (i.e., correlation between light-curve decline and luminosity), similar to what is found among normal SNe Ia (Phillips et al. 1999; Garnavich et al. 2004; Taubenberger et al. 2008). However, there are notable exceptions in the literature of $\mathrm{SNe}$ that share superficial similarities to SN 1991bg, but have unique properties not seen in the broader SN 1991bg subclass. Here, we will compare and contrast SN 2002es with known peculiar SN 1991bg-like SNe.

Foley et al. (2010b) found that SN 2006bt had broad, slowly evolving light optical curves $\left(\Delta m_{15}(B)=1.09 \pm 0.06 \mathrm{mag}\right)$ typical of a luminous event, but spectra more similar to those of 
Table 6

Comparison of Properties of Subluminous SN 1991bg-like Objects

\begin{tabular}{lccccc}
\hline \hline SN & $\begin{array}{c}M_{B} \\
(\mathrm{mag})\end{array}$ & $\begin{array}{c}\Delta m_{15}(B) \\
(\mathrm{mag})\end{array}$ & $\begin{array}{c}v_{\text {phot }}{ }^{\mathrm{a}} \\
\left(\mathrm{km} \mathrm{s}^{-1}\right)\end{array}$ & $\mathcal{R}(\mathrm{Si})$ & Source \\
\hline SN 1991bg & $-16.60 \pm 0.03$ & $1.93 \pm 0.10$ & 10,100 & $0.62 \pm 0.05$ & Taubenberger et al. (2008) \\
SN 2006bt & $-18.83 \pm 0.06$ & $1.09 \pm 0.06$ & 10,500 & $0.44 \pm 0.05$ & Foley et al. (2010b) \\
PTF 09dav & $-15.33 \pm 0.08$ & $1.87 \pm 0.06$ & 6100 & $0.35 \pm 0.05$ & Sullivan et al. (2011b) \\
PTF 10ops & $-17.66 \pm 0.06$ & $1.12 \pm 0.06$ & 10,000 & $0.58 \pm 0.06$ & Maguire et al. (2011) \\
SN 2002es & $-17.78 \pm 0.12$ & $1.28 \pm 0.04$ & 6000 & $0.55 \pm 0.05$ & This work \\
\hline
\end{tabular}

Note. ${ }^{a}$ As measured by the minimum in the absorption feature attributed to Si II $\lambda 6355$.

a cooler, subluminous event. A spectrum of the host galaxy from the SDSS (Abazajian et al. 2009) shows no signs of emission lines, indicating no recent star formation. Coupled with the position of SN 2006bt in the halo of the galaxy, the authors concluded that the progenitor of SN 2006bt was likely a low-mass star. Assuming no host-galaxy extinction, the peak absolute magnitude of SN 2006bt was $M_{B}=-18.94 \pm$ 0.06 mag, slightly less luminous than normal SNe Ia, but not a significant outlier in the Phillips relation. SN 2006bt had a lightcurve width similar to that of SN 2002es, but is significantly brighter. Unlike SN 2002es, the late-time decay in the light curves of SN $2006 \mathrm{bt}$ is consistent with ${ }^{56} \mathrm{Ni}$ decay. SN $2006 \mathrm{bt}$ had faster expansion velocities at maximum light compared to SN 2002es.

PTF has been a prolific discovery engine for unique transient events. Two subluminous $\mathrm{SNe}$, in particular, may be related to SN 2002es: PTF 09dav (Sullivan et al. 2011b) and PTF 10ops (Maguire et al. 2011). Both events have maximum-light spectra that resemble those of SN 1991bg. PTF 09dav had slow expansion velocities of $\sim 6100 \mathrm{~km} \mathrm{~s}^{-1}$ (similar to those of SN 2002es), while PTF 10ops had more typical velocities of $\sim 10,000 \mathrm{~km} \mathrm{~s}^{-1}$. Photometrically, both PTF 10ops and SN 2002es have broad light curves despite being subluminous. Both objects occupy a similar position off of the Phillips relation in Figure 3. PTF 09dav has a more typical SN 1991bg-like lightcurve shape $\left(\Delta m_{15}(B)=1.87 \pm 0.06 \mathrm{mag}\right)$, but was $\sim 1.5 \mathrm{mag}$ fainter than typical subluminous SNe Ia $\left(M_{B}=-15.5 \mathrm{mag}\right)$. However, SN 2002es is the only object where the optical light curves plummet after $t>+30$ days.

In Table 6, we summarize the photometric and spectroscopic properties of other peculiar SN 1991bg-like SNe in comparison to SN 2002es. While SN 2002es shares some characteristics with each of these SNe, no single previously published object is exactly like SN 2002es.

\subsection{Models}

Explaining the properties of SN 2002es within the confines of current models is challenging. Based on the star formation history of the host galaxy of SN 2002es (Neill et al. 2009), we expect the progenitor to be from a relatively old stellar population, indicating that it was an explosion of either a lowmass single star or a WD. In Section 4.5, we examined the possibility that SN 2002es is the result of core collapse of a lowmass single star. In this section, we explore possible models to explain the observed properties of SN 2002es assuming a WD progenitor.

The ".Ia" model has been proposed to explain rapidly evolving, subluminous thermonuclear events. They are the result of helium accretion onto a WD from a double-degenerate WD AM Canum Venaticorum (AM CVn) binary system (Bildsten et al.
2007; Shen \& Bildsten 2009; Shen et al. 2010) leading to a thermonuclear explosion of the accreted He envelope. These events are expected to have both a luminosity and a timescale that is one-tenth that of SNe Ia. Calculations of observable properties by Shen et al. (2010) find that the rise time of these objects is $<10$ days, with a fast decline after maximum. SN 2002es, on the other hand, has a rather broad light curve, with a rise that is likely longer than 10 days based on a first detection by LOSS of 9 days before the time of bolometric maximum light. The ejecta velocities are also expected to be $\sim 10^{4} \mathrm{~km} \mathrm{~s}^{-1}$, almost a factor of two larger than what is observed in SN 2002es.

Woosley \& Kasen (2011) studied a broader set of one-dimensional simulations with models involving a subChandrasekhar-mass carbon-oxygen (CO) WD undergoing helium accretion, including models that explode just the helium envelope and models that explode the entire star. The authors survey the parameter space of WD masses, accretion rates, and initial WD luminosities to produce model spectra and light curves for the resulting transient.

Models in which only the helium shell explodes (either through detonation or deflagration) have rise times $<10$ days and $\Delta m_{15}(B)>2.0 \mathrm{mag}$. The fast evolution of the $B$ band is caused by the small ejecta mass (depending on the size of the envelope) and the redistribution of flux from the optical to the NIR by IGEs in the ejecta (Kasen 2006). Detonations of "cold" WDs $\left(L=0.01 L_{\odot}\right)$ produce spectra lacking IMEs, which are clearly seen in our spectra of SN 2002es. One of the more promising models from the set of helium-envelope explosions involved "hot" WDs $\left(L=1 L_{\odot}\right)$. These explosions produce a significant amount of IMEs, resulting in spectra resembling SN 1991bg with ejecta velocities $\sim 9000 \mathrm{~km} \mathrm{~s}^{-1}$. These models produce a very small amount of ${ }^{56} \mathrm{Ni}\left(\sim 10^{-4} M_{\odot}\right)$ and are instead powered by ${ }^{48} \mathrm{Cr}$, producing a light curve with a three-day rise time and peak absolute magnitude of -13 . A light curve powered by ${ }^{48} \mathrm{Cr}$ and subsequent decay to ${ }^{48} \mathrm{~V}$ (half-life $\tau=16$ days) is an appealing explanation for the rapid fading of SN 2002es at $t>+30$ days; however, these models evolve too fast and have ejecta velocities that are inconsistent with what we observe.

Deflagrations within the helium envelope led to incomplete burning, producing lower ejecta velocities $\left(\sim 4000 \mathrm{~km} \mathrm{~s}^{-1}\right)$ and broader light curves caused by the increased diffusion time in comparison to the detonation model of comparable brightness. The light curves of these models are also powered by the decay of ${ }^{48} \mathrm{Cr}$, producing faint transients $\left(M_{B} \approx-14 \mathrm{mag}\right)$. However, the evolution of these light curves is still much too fast compared to $\mathrm{SN}$ 2002es.

Outcomes from exploding the entire star generally depended on the initial luminosity of the WD. "Cold" WDs required a larger accreted mass to ignite the helium envelope and drive an explosion of the entire star. As a result, the "cold" models 
contained an outer envelope of IGEs (synthesized from helium) which acted as a heating source to ionize IMEs and reduce their opacity. Model spectra of these events lack Si II, S II, and Ca II, all of which are present in SN 2002es. The "hot" models, on the other hand, explode with a smaller accreted envelope, allowing IMEs to appear in the spectra. In fact, the synthetic spectra for these objects look remarkably like those of normal SNe Ia and even produce a width-luminosity relation, similar to the Phillips relation, but with a different slope. However, the minimum expansion velocities seen in these models are $\sim 11,000 \mathrm{~km} \mathrm{~s}^{-1}$, much higher than what is observed for SN 2002es.

Pakmor et al. (2010, 2011) find in simulations that the merger of nearly-equal-mass WDs leads to underluminous explosions similar to SN 1991bg. Their model follows the evolution and subsequent explosion of two WDs of equal mass $\left(0.89 M_{\odot}\right)$. They find explosions that have roughly the same kinetic energy as a normal SN Ia $\left(\sim 10^{51} \mathrm{erg} \mathrm{s}^{-1}\right)$, but lower velocities due to the larger ejecta mass. Less ${ }^{56} \mathrm{Ni}\left(0.1 M_{\odot}\right)$ is synthesized due to lower densities in the final merged object, resulting in a subluminous event. Synthetic light curves of their models are similar to those of SN $1991 \mathrm{bg}$, but broader, with $\Delta m_{15}(B)=$ 1.4-1.7 mag. Synthetic spectra show strong titanium, as well as the presence of IMEs such as $\mathrm{Si}$ II and $\mathrm{O}_{\mathrm{I}}$ at velocities lower than typically seen in SN 1991bg-like objects. All of these match characteristics seen in SN 2002es. However, their models do not predict the fast drop in flux at $t>+30$ days, which is a key characteristic that makes SN 2002es a unique object.

\section{CONCLUSIONS}

SN 2002es is a peculiar, subluminous SN Ia with a unique combination of observables. At maximum light, spectra of SN 2002es are similar to the subluminous SN 1991bg, indicating a cool photosphere, but with ejecta velocity of $\sim 6000 \mathrm{~km} \mathrm{~s}^{-1}$. Such slow velocities are more characteristic of SN 2002cx-like objects. While also subluminous, SN 2002cx had a maximumlight spectrum resembling that of SN 1991T, which is characteristic of a hot photosphere.

Photometrically, SN 2002es has a broad light curve $\left(\Delta m_{15}(B)=1.28 \mathrm{mag}\right)$, despite being subluminous with a peak absolute magnitude of $M_{B}=-17.78 \mathrm{mag}$. SN 2002es is a $5 \sigma$ outlier in the Phillips relation (Phillips 1993; Phillips et al. 1999) used to calibrate the light-curve width versus luminosity relationship for SNe Ia. The $R$ - and $I$-band light curves are broad and lack the shoulder typically seen in SNe Ia.

Quantitative measurements of spectral features such as the silicon ratio $(\mathcal{R}(\mathrm{Si})$; Nugent et al. 1995) and the Si II $\lambda 6355$ velocity gradient are similar to those of SN 1991 bg-like objects. However, SN 2002es is an outlier in the usual relationships, which show strong correlations between these spectral measurements and light-curve parameters (Benetti et al. 2005; Branch et al. 2006).

From Arnett's law, we estimate a synthesized radioactive nickel mass of $0.17 M_{\odot}$ required to power the light curve. However, the bolometric light curve shows an unexpected drop in luminosity at $t>+30$ days. We are unable to fit the bolometric light curve with a toy model of the radiated luminosity that accounts for a decrease in the $\gamma$-ray trapping function as the ejecta expand homologously. If SN 2002es is a thermonuclear event, then the ejecta became optically thin to $\gamma$-rays in an unexpectedly dramatic fashion. Similarly, we are unable to explain the rapid decay by invoking dust formation or the IRC
(Axelrod 1980). Alternatively, SN 2002es could be powered by some other mechanism not yet understood.

SN 2002es exploded in the outskirts of the early-type S0 galaxy UGC 2708. SED fitting from Neill et al. (2009) and emission-line diagnostics indicate that UGC 2708 is likely a LINER galaxy with no current star formation. This points to an old star, likely a WD, as the progenitor to SN 2002es.

Finding a published model that matches the peculiar collection of observables found in SN 2002es is particularly challenging. We have not found a convincing match by comparing SN 2002es to Woosley \& Kasen (2011) models of WDs undergoing helium accretion from a companion star. Models of the merging and subsequent detonation of two equal-mass WDs from Pakmor et al. (2010, 2011) are promising, but do not reproduce the drop in luminosity one month after maximum light.

Looking through the existing LOSS photometry (Ganeshalingam et al. 2010) and the Berkeley Supernova Ia Program spectral database (Silverman et al. 2012b), we identify SN 1999bh as a probable SN 2002es-like event. Spectra and photometry of the object, while limited, match many of the observed characteristics of SN 2002es. Both objects are included in the LOSS SN rate studies (Leaman et al. 2011; Li et al. 2011a, 2011b), allowing us to estimate that roughly $3 \%$ of SNe Ia should be SN 2002es-like SNe within a fixed volume.

Ongoing surveys for transient objects, such as PTF and PanSTARRS, are likely to find more objects resembling SN 2002es. PTF has already published results for two peculiar subluminous objects that share some similarities to SN 2002es but also have distinct differences. We expect that future data sets will shed light on understanding the bizarre nature of these subluminous objects.

We are grateful to the Lick Observatory staff for their assistance with the operation of KAIT. We also thank B. Barris, P. Berlind, S. Jha, M. Papenkova, and B. Swift for their help with some of the observations. M.G. acknowledges useful and insightful conversations with D. Kasen and I. Kleiser regarding theoretical models of SNe Ia. M.G. is very grateful to S. Blondin for providing spectra of SN 2002es and SN 1999bh prior to publication of the CfA SN Ia spectroscopy paper. P.A.M. thanks G. Williams and P. Watje for late-epoch observations of SN 2002es.

The research of A.V.F.'s supernova group at UC Berkeley has been generously supported by the U.S. National Science Foundation (NSF; most recently through grants AST-0607485 and AST-0908886), the TABASGO Foundation, U.S. Department of Energy SciDAC grant DE-FC02-06ER41453, and U.S. Department of Energy grant DE-FG02-08ER41563. KAIT and its ongoing operation were made possible by donations from Sun Microsystems, Inc., the Hewlett-Packard Company, AutoScope Corporation, Lick Observatory, the NSF, the University of California, the Sylvia \& Jim Katzman Foundation, the Richard and Rhoda Goldman Fund, and the TABASGO Foundation. Supernova research at Harvard is supported in part by NSF grant AST-0907903. Some of the data presented in this paper were obtained at the W. M. Keck Observatory, which is operated as a scientific partnership among the California Institute of Technology, the University of California, and the National Aeronautics and Space Administration (NASA); the observatory was made possible by the generous financial support of the W. M. Keck Foundation. The authors wish to recognize and acknowledge the very significant cultural role and reverence that the summit of Mauna Kea has always had within the indigenous Hawaiian 
community; we are most fortunate to have the opportunity to conduct observations from this mountain. We made use of the NASA/IPAC Extragalactic Database (NED), which is operated by the Jet Propulsion Laboratory, California Institute of Technology, under contract with NASA.

\section{REFERENCES}

Abazajian, K. N., Adelman-McCarthy, J. K., Agüeros, M. A., et al. 2009, ApJS, 182,543

Aldering, G., Nugent, P., Ellis, R., Perlmutter, S., \& Folha, D. 1999, IAU Circ., 7138,3

Amanullah, R., Lidman, C., Rubin, D., et al. 2010, ApJ, 716, 712

Arnett, W. D. 1982, ApJ, 253, 785

Axelrod, T. S. 1980, PhD thesis, California Univ., Santa Cruz

Bailey, S., Aldering, G., Antilogus, P., et al. 2009, A\&A, 500, L17

Barbon, R., Benetti, S., Rosino, L., Cappellaro, E., \& Turatto, M. 1990, A\&A, 237, 79

Benetti, S., Cappellaro, E., Mazzali, P. A., et al. 2005, ApJ, 623, 1011

Bildsten, L., Shen, K. J., Weinberg, N. N., \& Nelemans, G. 2007, ApJ, 662, L95

Blondin, S., Mandel, K. S., \& Kirshner, R. P. 2011, A\&A, 526, A81

Blondin, S., Prieto, J. L., Patat, F., et al. 2009, ApJ, 693, 207

Branch, D., Dang, L. C., Hall, N., et al. 2006, PASP, 118, 560

Cardelli, J. A., Clayton, G. C., \& Mathis, J. S. 1989, ApJ, 345, 245

Chornock, R., Filippenko, A. V., Branch, D., et al. 2006, PASP, 118, 722

Chornock, R., Swift, B., Filippenko, A. V., \& Li, W. D. 2002, IAU Circ., 7965, 2

Conley, A., Sullivan, M., Hsiao, E. Y., et al. 2008, ApJ, 681, 482

de Vaucouleurs, G., de Vaucouleurs, A., Corwin, H. G., Jr., et al. 1991, in Third Reference Catalogue of Bright Galaxies, ed. G. de Vaucouleurs et al. (New York: Springer), 267

Fabricant, D., Cheimets, P., Caldwell, N., \& Geary, J. 1998, PASP, 110, 79

Filippenko, A. V. 1982, PASP, 94, 715

Filippenko, A. V. 1997, ARA\&A, 35, 309

Filippenko, A. V. 2003, in From Twilight to Highlight: The Physics of Supernovae, ed. W. Hillebrandt \& B. Leibundgut (Berlin: Springer), 171

Filippenko, A. V., Li, W. D., Treffers, R. R., \& Modjaz, M. 2001, in ASP Conf Ser. 246, Small Telescope Astronomy on Global Scales, ed. B. Paczyński, W.-P. Chen, \& C. Lemme (San Francisco, CA: ASP), 121

Filippenko, A. V., Richmond, M. W., Branch, D., et al. 1992, AJ, 104, 1543

Fisher, A., Branch, D., Nugent, P., \& Baron, E. 1997, ApJ, 481, L89

Folatelli, G., Phillips, M. M., Morrell, N., et al. 2012, ApJ, 745, 74

Foley, R. J., Brown, P. J., Rest, A., et al. 2010a, ApJ, 708, L61

Foley, R. J., Chornock, R., Filippenko, A. V., et al. 2009, AJ, 138, 376

Foley, R. J., Filippenko, A. V., \& Jha, S. W. 2008, ApJ, 686, 117

Foley, R. J., \& Kasen, D. 2011, ApJ, 729, 55

Foley, R. J., Narayan, G., Challis, P. J., et al. 2010b, ApJ, 708, 1748

Foley, R. J., Papenkova, M. S., Swift, B. J., et al. 2003, PASP, 115, 1220

Foley, R. J., Sanders, N. E., \& Kirshner, R. P. 2011, ApJ, 742, 89

Ganeshalingam, M., Li, W., Filippenko, A. V., et al. 2010, ApJS, 190, 418

Ganeshalingam, M., Li, W., \& Filippenko, A. V. 2011, MNRAS, 416, 2607

Garnavich, P. M., Bonanos, A. Z., Krisciunas, K., et al. 2004, ApJ, 613, 1120

Gerardy, C. L., Meikle, W. P. S., Kotak, R., et al. 2007, ApJ, 661, 995

Guy, J., Astier, P., Baumont, S., et al. 2007, A\&A, 466, 11

Heckman, T. M. 1980, A\&A, 87, 152

Hicken, M., Challis, P., Jha, S., et al. 2009a, ApJ, 700, 331

Hicken, M., Garnavich, P. M., Prieto, J. L., et al. 2007, ApJ, 669, L17

Hicken, M., Wood-Vasey, W. M., Blondin, S., et al. 2009b, ApJ, 700, 1097

Horne, K. 1986, PASP, 98, 609

Howell, D. A. 2001, ApJ, 554, L193

Howell, D. A., Sullivan, M., Brown, E. F., et al. 2009, ApJ, 691, 661

Howell, D. A., Sullivan, M., Nugent, P. E., et al. 2006, Nature, 443, 308

Jha, S., Riess, A. G., \& Kirshner, R. P. 2007, ApJ, 659, 122

Kasen, D. 2006, ApJ, 649, 939

Kasliwal, M. M., Kulkarni, S. R., Gal-Yam, A., et al. 2010, ApJ, 723, L98

Kasliwal, M. M., Kulkarni, S. R., \& Gal-Yam, A. 2011, arXiv:1111.6109

Kelly, P. L., Hicken, M., Burke, D. L., Mandel, K. S., \& Kirshner, R. P. 2010, ApJ, 715, 743

Kessler, R., Becker, A. C., Cinabro, D., et al. 2009, ApJS, 185, 32

Lampeitl, H., Smith, M., Nichol, R. C., et al. 2010, ApJ, 722, 566

Landolt, A. U. 1992, AJ, 104, 340

Landolt, A. U. 2009, AJ, 137, 4186

Law, N. M., Kulkarni, S. R., Dekany, R. G., et al. 2009, PASP, 121, 1395
Leaman, J., Li, W., Chornock, R., \& Filippenko, A. V. 2011, MNRAS, 412, 1419

Leibundgut, B. 2000, A\&AR, 10, 179

Leibundgut, B., Kirshner, R. P., Phillips, M. M., et al. 1993, AJ, 105, 301

Leloudas, G., Stritzinger, M. D., Sollerman, J., et al. 2009, A\&A, 505, 265

Li, W. 1999, IAU Circ., 7135, 2

Li, W., Chornock, R., Leaman, J., et al. 2011a, MNRAS, 412, 1473

Li, W., Filippenko, A. V., Chornock, R., \& Jha, S. 2003a, PASP, 115, 844

Li, W., Filippenko, A. V., Chornock, R., et al. 2003b, PASP, 115, 453

Li, W., Leaman, J., Chornock, R., et al. 2011b, MNRAS, 412, 1441

Li, W., Swift, B., \& Ganeshalingam, M. 2002, IAU Circ., 7959, 1

Maguire, K., Sullivan, M., Thomas, R. C., et al. 2011, MNRAS, 418, 747

Matheson, T., Challis, P., Kirshner, R., Berlind, P., \& Filippenko, A. V. 2002, IAU Circ., 7965, 3

Matheson, T., Kirshner, R. P., Challis, P., et al. 2008, AJ, 135, 1598

McClelland, C. M., Garnavich, P. M., Galbany, L., et al. 2010, ApJ, 720, 704

Miller, J. S., \& Stone, R. P. S. 1993, Lick Observatory Technical Report 66 (Santa Cruz: Lick Obs.)

Monnier Ragaigne, D., van Driel, W., Schneider, S. E., Balkowski, C., \& Jarrett, T. H. 2003, A\&A, 408, 465

Nadyozhin, D. K. 1994, ApJS, 92, 527

Narayan, G., Foley, R. J., Berger, E., et al. 2011, ApJ, 731, L11

Neill, J. D., Sullivan, M., Howell, D. A., et al. 2009, ApJ, 707, 1449

Nomoto, K., Filippenko, A. V., \& Shigeyama, T. 1990, A\&A, 240, L1

Nozawa, T., Maeda, K., Kozasa, T., et al. 2011, ApJ, 736, 45

Nugent, P., Kim, A., \& Perlmutter, S. 2002, PASP, 114, 803

Nugent, P., Phillips, M., Baron, E., Branch, D., \& Hauschildt, P. 1995, ApJ, 455, L147

Oke, J. B., Cohen, J. G., Carr, M., et al. 1995, PASP, 107, 375

Pakmor, R., Hachinger, S., Röpke, F. K., \& Hillebrandt, W. 2011, A\&A, 528, A117

Pakmor, R., Kromer, M., Röpke, F. K., et al. 2010, Nature, 463, 61

Parrent, J. T., Thomas, R. C., Fesen, R. A., et al. 2011, ApJ, 732, 30

Paxton, B., Bildsten, L., Dotter, A., et al. 2011, ApJS, 192, 3

Perets, H. B., Gal-Yam, A., Mazzali, P. A., et al. 2010, Nature, 465, 322

Perlmutter, S., Aldering, G., Goldhaber, G., et al. 1999, ApJ, 517, 565

Phillips, M. M. 1993, ApJ, 413, L105

Phillips, M. M., Lira, P., Suntzeff, N. B., et al. 1999, AJ, 118, 1766

Phillips, M. M., Li, W., Frieman, J. A., et al. 2007, PASP, 119, 360

Pinto, P. A., \& Eastman, R. G. 2000a, ApJ, 530, 744

Pinto, P. A., \& Eastman, R. G. 2000b, ApJ, 530, 757

Poznanski, D., Ganeshalingam, M., Silverman, J. M., \& Filippenko, A. V. 2011, MNRAS, 415, L81

Riess, A. G., Filippenko, A. V., Challis, P., et al. 1998, AJ, 116, 1009

Riess, A. G., Macri, L., Casertano, S., et al. 2011, ApJ, 732, 129

Scalzo, R. A., Aldering, G., Antilogus, P., et al. 2010, ApJ, 713, 1073

Schlegel, D. J., Finkbeiner, D. P., \& Davis, M. 1998, ApJ, 500, 525

Shen, K. J., \& Bildsten, L. 2009, ApJ, 699, 1365

Shen, K. J., Kasen, D., Weinberg, N. N., Bildsten, L., \& Scannapieco, E. 2010, ApJ, 715, 767

Silverman, J. M., \& Filippenko, A. V. 2012, MNRAS, submitted (arXiv:1202.3788)

Silverman, J. M., Ganeshalingam, M., Li, W., \& Filippenko, A. V. 2012a, MNRAS, submitted (arXiv:1202.2130)

Silverman, J. M., Ganeshalingam, M., Li, W., et al. 2011, MNRAS, 410, 585

Silverman, J. M., Kong, J. J., \& Filippenko, A. V. 2012b, MNRAS, submitted (arXiv:1202.2129)

Smith, N., Foley, R. J., \& Filippenko, A. V. 2008, ApJ, 680, 568

Sollerman, J., Holland, S. T., Challis, P., et al. 2002, A\&A, 386, 944

Sollerman, J., Lindahl, J., Kozma, C., et al. 2004, A\&A, 428, 555

Stanishev, V., Goobar, A., Benetti, S., et al. 2007, A\&A, 469, 645

Stritzinger, M., \& Leibundgut, B. 2005, A\&A, 431, 423

Stritzinger, M., Leibundgut, B., Walch, S., \& Contardo, G. 2006a, A\&A, 450, 241

Stritzinger, M., Mazzali, P. A., Sollerman, J., \& Benetti, S. 2006b, A\&A, 460, 793

Sullivan, M., Conley, A., Howell, D. A., et al. 2010, MNRAS, 406, 782

Sullivan, M., Guy, J., Conley, A., et al. 2011a, ApJ, 737, 102

Sullivan, M., Kasliwal, M. M., Nugent, P. E., et al. 2011b, ApJ, 732, 118

Suzuki, N., Rubin, D., Lidman, C., et al. 2012, ApJ, 746, 85

Taubenberger, S., Benetti, S., Childress, M., et al. 2011, MNRAS, 412, 2735

Taubenberger, S., Hachinger, S., Pignata, G., et al. 2008, MNRAS, 385, 75

Thomas, R. C., Aldering, G., Antilogus, P., et al. 2011, ApJ, 743, 27 
Turatto, M., Benetti, S., \& Cappellaro, E. 2003, in From Twilight to Highlight: The Physics of Supernovae, ed. W. Hillebrandt \& B. Leibundgut (Berlin: Springer), 200

Turatto, M., Benetti, S., Cappellaro, E., et al. 1996, MNRAS, 283, 1 Valenti, S., Pastorello, A., Cappellaro, E., et al. 2009, Nature, 459, 674 van den Bergh, S., Li, W., \& Filippenko, A. V. 2002, PASP, 114, 820

Wade, R. A., \& Horne, K. 1988, ApJ, 324, 411
Wang, X., Filippenko, A. V., Ganeshalingam, M., et al. 2009a, ApJ, 699, L139

Wang, X., Li, W., Filippenko, A. V., et al. 2008, ApJ, 675, 626

Wang, X., Li, W., Filippenko, A. V., et al. 2009b, ApJ, 697, 380

Wood-Vasey, W. M., Miknaitis, G., Stubbs, C. W., et al. 2007, ApJ, 666, 694

Woosley, S. E., \& Kasen, D. 2011, ApJ, 734, 38

Yamanaka, M., Kawabata, K. S., Kinugasa, K., et al. 2009, ApJ, 707, L118 